\title{
Dynamic Interaction between Cortico-Brainstem Pathways during Training-Induced Recovery in Stroke Model Rats
}

\author{
Akimasa Ishida, ${ }^{1}$ Kenta Kobayashi, ${ }^{2}$ Yoshitomo Ueda, ${ }^{1}$ Takeshi Shimizu, ${ }^{1}$ Naoki Tajiri, ${ }^{1}$ Tadashi Isa, ${ }^{3,4}$ \\ and ${ }^{\circ H i d e k i ~ H i d a}{ }^{1}$ \\ ${ }^{1}$ Department of Neurophysiology and Brain Science, Nagoya City University Graduate School of Medicine, Nagoya 467-8601, Japan, ${ }^{2}$ Section of Viral Vector \\ Development, National Institute for Physiological Sciences, Okazaki 444-8585, Japan, ${ }^{3}$ Department of Neuroscience, Graduate School of Medicine and \\ Faculty of Medicine, Kyoto University, Kyoto 606-8501, Japan, and ${ }^{4}$ Institute for the Advanced Study of Human Biology, Kyoto University, Kyoto \\ 606-8501, Japan
}

Reorganization of residual descending motor circuits underlies poststroke recovery. We previously clarified a causal relationship between the cortico-rubral tract and intensive limb use-induced functional recovery after internal capsule hemorrhage (ICH). However, other descending tracts, such as the cortico-reticular tract, might also be involved in rehabilitation-induced compensation. To investigate whether rehabilitation-induced recovery after ICH involves a shift in the compensatory circuit from the cortico-rubral tract to the cortico-reticular tract, we established loss of function of the cortico-rubral tract or/and cortico-reticular tract using two sets of viral vectors comprising the Tet-on system and designer receptors exclusively activated by the designer drug system. We used an ICH model that destroyed almost $60 \%$ of the corticofugal fibers. Anterograde tracing in rehabilitated rats revealed abundant sprouting of axons from the motor cortex in the red nucleus but not in the medullary reticular formation during the early phase of recovery. This primary contribution of the cortico-rubral tract was demonstrated by its selective blockade, whereas selective cortico-reticular tract silencing had little effect. Interestingly, cortico-rubral tract blockade from the start of rehabilitation induced an obvious increase of axon sprouting in the reticular formation with substantial functional recovery. Additional cortico-reticular tract silencing under the cortico-rubral tract blockade significantly worsened the recovered forelimb function. Furthermore, the alternative recruitment of the cortico-reticular tract was gradually induced by intensive limb use under cortico-rubral tract blockade, in which cortico-reticular tract silencing caused an apparent motor deficit. These findings indicate that individual cortico-brainstem pathways have dynamic compensatory potency to support rehabilitative functional recovery after ICH.

Key words: internal capsule; intracerebral hemorrhage; red nucleus; rehabilitation; reorganization; reticular formation

Significance Statement

This study aimed to clarify the interaction between the cortico-rubral and the cortico-reticular tract during intensive rehabilitation and functional recovery after capsular stroke. Pathway-selective disturbance by two sets of viral vectors revealed that the cortico-rubral tract was involved in rehabilitation-induced recovery of forelimb function from an early phase after internal capsule hemorrhage, but that the cortico-reticular tract was not. The sequential disturbance of both tracts revealed that the cortico-reticular tract was recruited and involved in rehabilitation-induced recovery when the cortico-rubral tract failed to function. Our data demonstrate a dynamic compensatory action of individual cortico-brainstem pathways for recovery through poststroke rehabilitation.

\section{Introduction}

Reorganization of motor pathways, such as unmasking of silent pathways or generation of new synaptic connections by axonal

Received March 22, 2019; revised July 22, 2019; accepted July 31, 2019.

Author contributions: A.I., T.I., and H.H. designed research; A.I. performed research; A.I. analyzed data; A.I. wrote the first draft of the paper; A.I., Y.U., T.S., N.T., T.I., and H.H. edited the paper; A.I., T.I., and H.H. wrote the paper; K.K. contributed unpublished reagents/analytic tools.

This work was supported by Japan Society for the Promotion of Science Grants-in-Aid for Scientific Research 15 K16361 and 18K10718 to A.I., Ministry of Education, Culture, Sports, Science and Technology Grant-in-Aid for Scientific Research on Innovative Areas (Adaptive Circuit Shift, 15H01445) to H.H., and the Takeda Science Founda- sprouting, has been considered as the underlying mechanism for functional recovery and is a key factor in poststroke rehabilitation (Taub et al., 2002; Murphy and Corbett, 2009). Various forms of motor circuit reorganization after lesion of the primary motor

tion. We thank Kaoru Isa and Kota Tokuoka for technical support; and Jeremy Allen and Sydney Koke (Edanz Group; www.edanzediting.com/ac) for editing a draft of this manuscript.

The authors declare no competing financial interests.

Correspondence should be addressed to Hideki Hida at hhida@med.nagoya-cu.ac.jp.

https://doi.org/10.1523/JNEUROSCI.0649-19.2019

Copyright $\odot 2019$ the authors 
cortex or the cortico-spinal tract (CST) have been demonstrated, such as recruitment of premotor cortex (Liu and Rouiller, 1999; Fridman et al., 2004), sprouting from the contralateral CST (Bareyre et al., 2004; Maier et al., 2008), and using alternative pathways, such as propriospinal neurons (Tohyama et al., 2017). The cortico-brainstem pathways may also be potential compensatory pathways after stroke.

The red nucleus is one of the brainstem nuclei that project their axons to the spinal cord, termed the rubrospinal tract. The cortico-rubral and rubrospinal tracts can mediate functional recovery after CST lesion (Z'Graggen et al., 1998; Mosberger et al., 2018). Using a pathway-selective blocking technique with viral vectors, we recently demonstrated a substantial increase in the number of cortico-rubral tract axons by intensive impaired forelimb use after capsular hemorrhage, revealing a causal relationship between the cortico-rubral tract and functional forelimb recovery (Ishida et al., 2016). These studies indicate that the cortico-rubral tract can be one of the key routes for cortexdependent reorganization of motor circuits in response to rehabilitation. However, several questions regarding the relationship between the cortico-rubral tract and functional recovery remain unanswered. The timing of when the cortico-rubral tract is recruited during the process of recovery is one of the issues to be explored. Elucidation of the precise role of the cortico-rubral tract in the process of regaining impaired functions is an important challenge for the development of poststroke rehabilitation.

In addition to the cortico-rubral tract, the cortico-reticular tract is also a potential target for poststroke rehabilitation: the medullary reticular nuclei gigantocellular reticular nucleus (Gi) and the medullary reticular nucleus ventral part (MdV) consist of neurons that project their axons bilaterally to cervical motoneurons (Peterson et al., 1979). Involvement of the MdV in skilled grasping movements in rodents was recently demonstrated (Esposito et al., 2014), and the importance of Gi and MdV neurons in the relay between the motor cortex and spinal cord after cortical stroke or spinal cord injury was also reported (Bachmann et al., 2014; Asboth et al., 2018). Thus, the cortico-reticular tract might be an important substrate for functional recovery after CNS injury. We previously demonstrated a causal link between the cortico-rubral tract and functional recovery; however, under the conditions used for the cortico-rubral tract blockade, the possibility of alternative tracts contributing to the recovery was assumed (Ishida et al., 2016). Therefore, we hypothesized that the cortico-reticular tract, in addition to the cortico-rubral tract, could be involved in rehabilitation-induced recovery after lesion of the CST. However, the relationship and interaction between these two cortico-brainstem pathways in functional recovery have not been explored.

In this study, we first investigated the timing of when the cortico-rubral tract is recruited during poststroke rehabilitation. We next investigated the possibility that the cortico-reticular tract is recruited during rehabilitation-induced recovery after stroke. For this purpose, using the internal capsule hemorrhage (ICH) rat model (Masuda et al., 2007; Ishida et al., 2011), the function of the cortico-rubral tract or the cortico-reticular tract was selectively manipulated during or after rehabilitation by a double viral vector technique, one expressing enhanced tetanus neurotoxin under the Tet-activator system and another expressing hM4D(Gi) under a Cre driver. We independently silenced the cortico-rubral tract and cortico-reticular tract to clarify the compensatory action between the two tracts during rehabilitationinduced functional recovery. Our study demonstrated that the cortico-rubral tract was directly linked to functional recovery induced by rehabilitation after ICH from the early-recovery stage. In addition, the cortico-reticular tract was rapidly recruited and involved in the recovery when the cortico-rubral tract failed to function.

\section{Materials and Methods}

Animals. Male Wistar rats (250-300 g) were group-housed under controlled temperature conditions $\left(22^{\circ} \mathrm{C}-23^{\circ} \mathrm{C}\right)$ and a $12 \mathrm{~h}$ light-dark cycle, with free access to food and water. Most of the experimental procedures were approved by the Committee of Animal Experimentation of Nagoya City University Medical School and were conducted in accordance with the animal care guidelines of Nagoya City University. A part of the experiments (Electrophysiological experiments under anesthesia) were conducted in the Graduate School of Medicine, under permission from the Committee of Animal Experimentation of Graduate School of Medicine, Kyoto University.

$I C H$. Rats were anesthetized with a mixture of ketamine $(70 \mathrm{mg} / \mathrm{kg}$ body weight, i.p., Fujita Pharmaceutical) and xylazine (10 mg/kg, i.p., Bayer), followed by atropine $(0.1 \mathrm{mg} / \mathrm{kg}$, i.p., Mitsubishi Tanabe Pharma) and dexamethasone $(0.1 \mathrm{mg} / \mathrm{kg}$, i.m., Aspen Pharmacare). Rats were set in a stereotaxic frame after shaving of fur from the scalp. The skull was exposed by incision of the skin along the midline and drilled to create a small hole at $3.8 \mathrm{~mm}$ lateral to the midline and $1.8 \mathrm{~mm}$ caudal to bregma contralateral to the preferred forelimb. A preferred limb was determined in training sessions of the single-pellet reaching test. Type IV collagenase (15 units/ml in saline, $1.4 \mu \mathrm{l}$, Merck) was injected using a glass micropipette (tip diameter: $50-60 \mu \mathrm{m}$ ) connected to a Hamilton syringe ( $5 \mathrm{~mm}$ below the brain surface, at a rate of $0.2 \mu \mathrm{l} / \mathrm{min}$ over $7 \mathrm{~min}$ ). The pipette was left in place for $7 \mathrm{~min}$ to prevent backflow. Sham groups received the same surgery and were injected with the equivalent amount of saline. After injection, the scalp was sutured and rats were allowed to recover in their home cage. One day after surgery, the extent of motor dysfunction was assessed according to the motor deficit score (MDS), as previously described (Masuda et al., 2007; Ishida et al., 2015). If the MDS was $<8$, the rat was excluded from the study. To quantify the lesioned fibers of the internal capsule, Klüver-Barrera staining was performed after completion of the experiments (see Fig. 1b). We measured the area of remaining fibers in the internal capsule after binarization. The damaged area of the internal capsule was calculated as percentage of the total internal capsule area and was normalized against that of the intact side.

Rehabilitation. We used forced impaired limb use as poststroke rehabilitation as described previously (Ishida et al., 2011, 2015). In brief, the unimpaired forelimb and upper torso of the rats were restrained by soft felt and plaster of Paris strips under isoflurane anesthesia ( $2 \%$ for induction, $1 \%$ for maintenance; Mylan). After casting, the rats were returned to their home cages and lived with the cast for $7 \mathrm{~d}$. The cast allowed subtle limb movement but prevented the use of limbs on the unimpaired side in all daily activities.

Anterograde tracing. At 1 or $30 \mathrm{~d}$ after ICH surgery, biotinylated dextran amine (BDA: MW 10,000; Thermo-Fisher Scientific) was injected into the motor cortex forelimb area of the injured side. Rats were anesthetized by ketamine $(70 \mathrm{mg} / \mathrm{kg})$ and xylazine $(10 \mathrm{mg} / \mathrm{kg})$ and set in a stereotaxic frame, and then BDA $(0.5 \mu \mathrm{l}, 5 \%$ in $0.1 \mathrm{M} \mathrm{PBS})$ was injected at four sites (axis to bregma AP $2.5 \mathrm{~mm}$, ML $2.0 \mathrm{~mm}$; AP $2.5 \mathrm{~mm}$, ML 3.0 $\mathrm{mm}$; AP $0.5 \mathrm{~mm}$, ML $2.5 \mathrm{~mm}$; and AP $0.5 \mathrm{~mm}$, ML $3.5 \mathrm{~mm}$ ) using a pulled glass capillary (diameter $40-50 \mu \mathrm{m}$ ) attached to a microliter Hamilton syringe at a flow rate of $100 \mathrm{nl} / \mathrm{min}$ controlled by an electric pump (ESP-64; Eicom). On day 12 and 51 after BDA injection, animals were perfused transcardially with $0.1 \mathrm{M}$ PBS and 4\% PFA under deep anesthesia with pentobarbital sodium ( $>100 \mathrm{mg} / \mathrm{kg}$, i.p., Kyoritsu Seiyaku), and their brains were dissected. BDA was injected into the motor cortex forelimb area in 4 naive rats. The rats were then perfused $12 \mathrm{~d}$ after the BDA injections. After postfixation and cryoprotection with $10 \%$, $20 \%$, and $30 \%$ sucrose in $0.1 \mathrm{M}$ PBS, $40-\mu \mathrm{m}$-thick coronal sections were cut using a cryostat (CM 1520, Leica Microsystems). The sections were rinsed in PBS several times and processed with $0.6 \% \mathrm{H}_{2} \mathrm{O}_{2}$ and $20 \%$ DMSO in methanol for $30 \mathrm{~min}$ to block endogenous peroxidase. After rinsing in $\mathrm{PBS}$, the sections were incubated in $\mathrm{PBS}$ containing $2 \% \mathrm{ABC}$ 
Elite reagent (VECTASTAIN Elite ABC; Vector Laboratories) and 0.3\% Triton X-100 for $2 \mathrm{~h}$. Sections were then rinsed in $10 \mathrm{~mm}$ TBS and incubated in a DAB-Ni reaction solution $(0.01 \% \mathrm{DAB}, 1.0 \%$ nickel ammonium sulfate, $0.0003 \% \mathrm{H}_{2} \mathrm{O}_{2}$ in TBS) for $30 \mathrm{~min}$. Then, the stained sections were counterstained by $0.5 \%$ neutral red (Wako Pure Chemical Industries), and dried and dehydrated before being coverslipped with Entellan new (Merck). Stained sections were observed and images recorded using light microscopy (Axioplan2, Carl Zeiss; AX70, Olympus). The number of BDA-positive bouton-like varicosities that were in contact with neurons in the magnocellular and parvocellular regions of the red nucleus ( $\mathrm{RNm}$ and $\mathrm{RNp}$ ), and the $\mathrm{Gi}$ and $\mathrm{MdV}$ were counted from four sections at $400 \times$ magnification. The total number of BDA-positive fibers in the cerebral peduncle was counted from three adjacent sections rostral to the red nucleus, averaged, and used to normalize the uptake efficacy of BDA and lesion size. The results are expressed as the mean number of bouton-like varicosities in RNp, RNm, Gi, and MdV divided by the mean number of fibers counted in the cerebral peduncle for each animal.

Preparation and injection of viral vectors. To silence the corticobrainstem pathways, we applied a double viral vector infection technique, as described previously (Kinoshita et al., 2012). The following virus vectors were used: fusion glycoprotein Type E (FuG-E)tetracycline-responsive element (TRE)-enhanced green fluorescent protein (EGFP)-enhanced tetanus neurotoxin light chain (eTeNT) (titer, $2.2-2.4 \times 10^{12}$ copies/ml), FuG-E-murine stem cell virus (MSCV)Cre (titer, $6.5 \times 10^{10}$ copies $/ \mathrm{ml}$ ), adeno-associated virus (AAV)-DJcytomegalovirus (CMV)-reverse tetracycline transactivator (rtTAV16) (titer, 5.8-6.8 $\times 10^{10}$ copies $/ \mathrm{ml}$ ), and AAV-DJ-elongation factor 1- $\alpha$ $(\mathrm{EF} 1 \alpha)$-DIO-hM4D(Gi)-mCherry (titer, $5.0 \times 10^{9}$ copies $/ \mathrm{ml}$ ). The FuG-E-pseudotyped vector was modified from a previously reported an HIV Type 1 (HIV-1)-based vector to produce enhanced efficiency of retrograde gene delivery (Kato et al., 2014; Kobayashi et al., 2017). FuGE-TRE-EGFP-eTeNT and AAV-DJ-CMV-rtTAV16, comprising the Tet-on system, were used to blockade the cortico-rubral tract. Doubleinfected cortico-rubral neurons expressed eTeNT, which depresses synaptic transmission during DOX administration. FuG-E-MSCV-Cre and AAV-DJ-EF1 $\alpha$-DIO-hM4D(Gi)-mCherry, comprising designer receptors exclusively activated by the designer drug (DREADD) system, were used to silence the double-infected cortico-reticular neurons.

Thirty-three days before ICH surgery, rats were anesthetized with ketamine $(70 \mathrm{mg} / \mathrm{kg})$ and xylazine $(10 \mathrm{mg} / \mathrm{kg})$ followed by administration of atropine $(0.1 \mathrm{mg} / \mathrm{kg})$ and dexamethasone $(0.1 \mathrm{mg} / \mathrm{kg})$ for premedication, and then mounted in a stereotaxic frame. For cortico-rubral tract blockade, FuG-E-TRE-EGFP-eTeNT was injected into the two sites of the red nucleus using a pulled glass micropipette $(40-50 \mu \mathrm{m}$ diameter) connected to a microsyringe and syringe pump. The coordinates for injections were as follows: AP $-5.3 \mathrm{~mm}, \mathrm{ML} 1.7 \mathrm{~mm}$, DV axis to the skull surface $8.6 \mathrm{~mm}$; AP $-5.8 \mathrm{~mm}$, ML $1.7 \mathrm{~mm}$, and DV $8.3 \mathrm{~mm}$. The micropipette was lowered at an angle of $5^{\circ}$ from the vertical axis to lateral to avoid the sinus. Each $0.4 \mu \mathrm{l}$ injection was performed at a rate of 0.1 $\mu \mathrm{l} / \mathrm{min}$ over $4 \mathrm{~min}$, and the needle was left in place for $4 \mathrm{~min}$ to prevent reflux. For silencing the cortico-reticular tract, bilateral injections of FuG-E-MSCV-Cre into the reticular formation, including Gi and MdV neurons, were conducted. Each animal had a total of 10 injections of 0.4 $\mu \mathrm{l}$ each spaced at $0.5 \mathrm{~mm}$ along the midline. The coordinates for injections were as follows: AP $-11.0 \mathrm{~mm}$, LM $0.8 \mathrm{~mm}$, DV $8.0 \mathrm{~mm}$; AP -11.5 $\mathrm{mm}$, LM $0.8 \mathrm{~mm}$, DV $7.6 \mathrm{~mm}$; AP $-12.0 \mathrm{~mm}$, LM $0.8 \mathrm{~mm}$, DV $7.3 \mathrm{~mm}$; AP $-12.5 \mathrm{~mm}$, LM $1.0 \mathrm{~mm}$, DV $7.3 \mathrm{~mm}$; and AP $-13.0 \mathrm{~mm}$, LM $1.0 \mathrm{~mm}$, DV $7.0 \mathrm{~mm}$, bilaterally.

Seven to $10 \mathrm{~d}$ after lentivirus injection, the forelimb area of the motor cortex was exposed by careful craniotomy under anesthesia. AAV-DJCMV-rtTAV16 $(0.4 \mu \mathrm{l})$ or AAV-DJ-EF1 $\alpha$-DIO-hM4D(Gi)-mCherry $(0.4 \mu \mathrm{l})$ was injected into the motor cortex at eight sites to disturb the function of the cortico-rubral tract or the cortico-reticular tract. The coordinates for injections were as follows: AP $1.0 \mathrm{~mm}$, ML $2.5 \mathrm{~mm}$; AP $1.0 \mathrm{~mm}$, ML $3.5 \mathrm{~mm}$; AP $1.5 \mathrm{~mm}$, ML $3.0 \mathrm{~mm}$; AP $2.0 \mathrm{~mm}$, ML $2.5 \mathrm{~mm}$; AP $2.0 \mathrm{~mm}$, ML $3.5 \mathrm{~mm}$; AP $2.5 \mathrm{~mm}$, ML $3.0 \mathrm{~mm}$; AP $3.0 \mathrm{~mm}$, ML 2.5 $\mathrm{mm}$; and AP $3.0 \mathrm{~mm}$, ML $3.5 \mathrm{~mm}$. Each injection was performed at 1.5 $\mathrm{mm}$ below the skull surface for $4 \mathrm{~min}$ at a rate of $0.1 \mu \mathrm{l} / \mathrm{min}$. In case of the sequential silencing of both tracts, an equal amount $(0.4 \mu \mathrm{l})$ of both vectors was mixed and injected into each site.

Administration of DOX and clozapine-N-oxide (CNO). For corticorubral tract blockade, doxycycline hyclate (DOX, Merck) was administered orally via drinking water $(3 \mathrm{mg} / \mathrm{ml}$ in $5 \%$ sucrose solution) and pellets $(100 \mathrm{mg} / \mathrm{kg})$. In addition, a single intraperitoneal injection of DOX $(10 \mu \mathrm{g} / \mathrm{g}$ body weight, in $0.9 \% \mathrm{NaCl})$ was given on the first day of oral DOX administration. For silencing the cortico-reticular tract, $\mathrm{CNO}$ (Enzo Life Sciences) was administered via intraperitoneal injection (3 $\mu \mathrm{g} / \mathrm{g}$ body weight, in $0.9 \% \mathrm{NaCl}) 20 \mathrm{~min}$ before testing. Behavioral assessments were conducted within 20-40 min after the CNO injection. The time courses of drug administration are presented in Figures $2 a, 3 a$, $6 a$, and $7 a$.

Behavioral tests. Single-pellet reaching task was performed as described previously (Metz and Whishaw, 2000). In brief, an experimental Plexiglas chamber $(45 \times 13 \times 40 \mathrm{~cm})$ had a narrow longitudinal slit in the front wall and a platform ( $3 \mathrm{~cm}$ wide, $4 \mathrm{~cm}$ from the bottom) was attached to the outside of the front wall. The platform has two indentations positioned $1.5 \mathrm{~cm}$ from the center of the slit in which sucrose pellets (45 mg; Bioserv) can be placed. Each rat was put in the apparatus and trained to retrieve the pellets placed in either indentation using their dominant limb, which was determined at the first training session. Food control was performed in the reaching task training and testing periods so that the animals neither gained nor lost weight. Each training session consisted of 20 trials and was performed once per day. All rats were trained for 3 weeks; and if a rat failed to retrieve more than eight pellets per session on the last three training sessions, it was excluded from the study. Baseline performances were measured 3-5 d before ICH surgery. The baseline and testing session consisted of 20 trials and was performed once per day. The time courses of the assessments are presented in Figure $2 a$, $3 a, 6 a$, and $7 a$. Reaching behaviors were videotaped ( 60 frames/s) from both side and front and were analyzed in detail. Averaged reaching distance from the front wall of the apparatus to the farthest point of each reach was measured and calculated using frame-by-frame analysis. In addition, averaged angle of wrist pronation during pellet grasping and subsequent supination during withdrawal was also analyzed from the frontal view.

Electrophysiological experiments. To examine the effect and time course of DREADD-based silencing of the cortico-reticular tract, electrophysiological experiments were conducted under anesthesia. Four weeks before the experiment, FuG-E-MSCV-Cre and AAV-DJ-EF1 $\alpha$-DIO$\mathrm{hM} 4 \mathrm{D}(\mathrm{Gi})$-mCherry were injected into intact rats following the method above. The anesthesia was introduced with a mixture of ketamine (70 $\mathrm{mg} / \mathrm{kg})$ and xylazine $(10 \mathrm{mg} / \mathrm{kg})$, and then atropine $(0.1 \mathrm{mg} / \mathrm{kg})$ and dexamethasone $(0.1 \mathrm{mg} / \mathrm{kg})$ were administered. Tracheal cannulation was performed, and artificial respiration was provided with a constant volume respirator (SN-480-7, Shinano Apparatus) while the animals were immobilized using pancuronium bromide $(0.2 \mathrm{mg} / \mathrm{kg}$, Myoblock; Merck Sharp and Dohme). The rectal temperature was maintained at $34^{\circ} \mathrm{C}-37^{\circ} \mathrm{C}$. Craniotomy of the occipital bone and laminectomy at $\mathrm{C} 1$ and $\mathrm{C} 2$ vertebrae were performed. Then the most caudal end of the cerebellum was aspirated to expose the obex and caudal brainstem. The dura mater was incised, and the exposed brainstem and spinal cord were covered by liquid paraffin to avoid drying. A small hole was made by drilling above the position of the cerebral peduncle of the lesioned hemisphere.

A bipolar tungsten electrode was inserted into the cerebral peduncle (AP $-6.7 \mathrm{~mm}$, ML $1.4 \mathrm{~mm}$, DV $8.7 \mathrm{~mm}$ ) to stimulate the corticofugal fibers. A train of three consecutive bipolar pulses $(180-200 \mu \mathrm{A}, 0.1 \mathrm{~ms}$ duration, $3.3 \mathrm{~ms}$ interval) was applied via the stimulating electrode from an electrically isolated, constant current stimulator (SEN-8203, Nihon Kohden). The interval between each train pulse was $5.1 \mathrm{~s}$. For the recording of local field potentials (LFPs), a tungsten electrode (100-200 k $\Omega$ impedance) was inserted into the caudal reticular formation $(1.0 \mathrm{~mm}$ anterior and $0.5 \mathrm{~mm}$ lateral from the obex, $1.0 \mathrm{~mm}$ depth from the surface) with a rostrally oriented angle of $30^{\circ}$. The descending spinal volley was recorded from the dorsal surface of the spinal cord, as cord dorsum potential (CDP), at the C1-C2 segment using a silver ball electrode. Once stable detection of LFP and CDP signals was established, recording was started. After baseline recording, $\mathrm{CNO}$ ( $3 \mathrm{mg} / \mathrm{kg}$ in saline) 
was administered via intraperitoneal injection, and the signals were recorded for $1 \mathrm{~h}$. Ketamine and pancuronium bromide were administered every $30 \mathrm{~min}$ to maintain anesthesia and immobilization. All signals were digitized and stored (Digidata 1440A; Molecular Devices), and analyzed off-line using Clampfit 10 (Molecular Devices).

Histological assessments. At the end of the experiments, the animals were perfused transcardially with 4\% PFA under deep anesthesia by sodium pentobarbital ( $>100 \mathrm{mg} / \mathrm{kg}$, Kyoritsu Seiyaku), and the brain was dissected and postfixed. After cryoprotection, $40 \mu \mathrm{m}$ coronal sections were cut on a cryostat. The sections were rinsed in PBS and treated with $0.6 \% \mathrm{H}_{2} \mathrm{O}_{2}$ and $20 \%$ DMSO in methanol for $30 \mathrm{~min}$. After rinsing in PBS, the sections were blocked in PBS containing $0.3 \%$ Triton X-100 and 10\% normal goat serum for $1 \mathrm{~h}$. Mouse anti-GFP antibody (1:1000, Nacalai Tesque) or rabbit anti-mCherry antibody (1:1000, Abcam) was applied overnight at $4^{\circ} \mathrm{C}$. After several washing steps, sections were incubated with biotinylated goat anti-mouse IgG antibody (1:200) or goat antirabbit IgG antibody (1:200) for $2 \mathrm{~h}$ at room temperature. After rinsing, the sections were incubated in ABC Elite reagent in PBS and 0.3\% Triton-X for $2 \mathrm{~h}$. The sections were then rinsed in TBS, and DAB-Ni reaction solution was applied for $30 \mathrm{~min}$. Then, sections were dried and dehydrated before being coverslipped with Entellan new. Every fifth section was selected, and GFP or mCherry-positive neurons were counted from 3.2 to $0.4 \mathrm{~mm}$ rostral to bregma using an Axioplan2 (Carl Zeiss) or AX70 microscope (Olympus). For immunofluorescence experiments, every 10th section was selected, blocked, and incubated with the primary antibodies described above. After rinsing, sections were incubated with a mixture of AlexaFluor-488 goat anti-mouse IgG antibody (1:200) and AlexaFluor-594 goat anti-rabbit IgG antibody (1:200) for $2 \mathrm{~h}$ at room temperature. Finally, sections were washed and mounted on glass slides. After drying overnight, the sections were coverslipped with ProLong Gold Antifade Mountant (Thermo-Fisher Scientific). Slides were observed using an Axioplan2 or an AX70 microscope (Olympus).

Experimental design and statistical analysis. In the present study, 3 rats did not meet the inclusion criteria for the skilled reaching task. Four rats died during surgery, and 3 rats were excluded because of insufficient motor dysfunction (MDS was $<8$ at $1 \mathrm{~d}$ after surgery). Three rats were excluded after histological assessment because the extent of viral infection was insufficient. All statistical analyses were conducted using JMP version10 (JMP statistical software; SAS Institute). The threshold for significance for all experiments was set at $p \leq 0.05$. Comparison of histological data was performed using Student's $t$ test. Behavioral assessments were analyzed by two-way repeated-measures ANOVA, and the comparison between groups at each time point was analyzed by Student's $t$ test. To detect differences within groups over time, Bonferroni correction for multiple comparisons was used. The number of animals used in each experiment can be found in Results. All data are plotted as mean \pm SEM.

\section{Results}

\section{Intensive rehabilitation training increases the cortico-rubral} connection in the early phase after capsular hemorrhage

We previously demonstrated a causal relationship between the cortico-rubral tract and functional recovery of an impaired forelimb by intensive impaired limb use at $1-8 \mathrm{~d}$ after capsular hemorrhage (Ishida et al., 2016). This was revealed by selective cortico-rubral tract blockade in the chronic phase following confirmation of an abundant increase in cortico-rubral axonal projections at day 51 after ICH. However, the effect of selective cortico-rubral tract blockade in the early phase has not been determined. We previously demonstrated that forced limb use at $1-8 \mathrm{~d}$ resulted in significant functional recovery as early as $12 \mathrm{~d}$ after ICH (Ishida et al., 2011, 2015, 2016). Therefore, we investigated the involvement of the cortico-rubral connection at this time point. We assumed that there would be an abundant increase in axonal projections to the red nucleus at day 12 after ICH and that a selective cortico-rubral tract blockade at this point would mask functional recovery induced by forced-limb use.
To clarify this possibility, we conducted anterograde tracing of axonal projections from the forelimb area of the ipsilesional motor cortex of ICH rats: BDA was injected into the ipsilesional motor cortex forelimb area at post-ICH days 1 and 30 (Fig. 1a). ICH disrupted most of the internal capsule (Fig. 1b), leading to severe and chronic motor deficit of the contralateral forelimb. ICH-REHAB group rats received forced impaired forelimb use rehabilitation by restraint of the intact limb (Fig. 1c). KlüverBarrera staining revealed that the percentage of damaged corticofugal fibers in the internal capsule was not significantly different between ICH and ICH-REHAB groups (ICH, $n=7$ : $59.2 \pm 7.9 \%$, ICH-REHAB, $n=8: 60.1 \pm 7.9 \%, p=0.973)$.

At day 12 (D12), more BDA-positive varicosities touching red nucleus neurons were present in the ICH-REHAB group (Fig. $1 g, g^{\prime}$ ) compared with the ICH group (Fig. $1 d, d^{\prime}$ ). Quantitative analysis revealed a significant increase in BDA-positive varicosities around the red nucleus in ICH-REHAB group $(n=6)$ compared with ICH control group $(n=6)$ at day $12(\mathrm{RNp}$, ICH: $0.13 \pm 0.07$ boutons/total fibers, ICH-REHAB: $0.42 \pm 0.04, p=$ 0.026; RNm, ICH: $0.08 \pm 0.06$, ICH-REHAB: $0.22 \pm 0.01, p=$ 0.049; Fig. 1j). We also observed a significant increase in BDAlabeled varicosities in the ICH-REHAB group $(n=7)$ compared with the ICH group $(n=5)$ at day 51 (D51, RNp, ICH: $0.32 \pm$ 0.14, ICH-REHAB: $0.75 \pm 0.12, p=0.043$; RNm, ICH: $0.20 \pm$ 0.07 , ICH-REHAB: $0.42 \pm 0.07, p=0.042$; Fig. $1 k$; data are quoted from Ishida et al., 2016).

To assess cortico-reticular tract involvement in rehabilitation-induced recovery after CST lesion, we also investigated the effects of intensive limb use on the cortico-reticular tract projections at D12 and D51. Although many BDA-positive fibers and varicosities were observed around the caudal reticular nuclei, Gi (Fig. $\left.1 e, e^{\prime}, h, h^{\prime}\right)$ and $\mathrm{MdV}$ (Fig. $\left.1 f, f^{\prime}, i, i^{\prime}\right)$, in both $\mathrm{ICH}(n=6)$ and ICH-REHAB $(n=6)$ groups, there was no significant difference between groups in any region at D12 (Gi-cont, ICH: $0.30 \pm 0.02$ boutons/total fibers, ICH-REHAB: $0.37 \pm 0.10, p=0.100$; Giipsi, ICH: $0.16 \pm 0.03$, ICH-REHAB: $0.20 \pm 0.04, p=0.149$; MdV-cont, ICH: $0.50 \pm 0.02$, ICH-REHAB: $0.64 \pm 0.22, p=$ 0.997; MdV-ipsi, ICH: $0.11 \pm 0.02$, ICH-REHAB: $0.16 \pm 0.02$, $p=0.144$; Fig. $1 j$ ). There was also no significant difference between ICH $(n=5)$ and ICH-REHAB $(n=7)$ groups at D51 (Gi-cont, ICH: $0.27 \pm 0.12$, ICH-REHAB: $0.50 \pm 0.09, p=0.118$; Gi-ipsi, ICH: $0.09 \pm 0.03$, ICH-REHAB: $0.16 \pm 0.03, p=0.114$; MdV-cont, ICH: $0.75 \pm 0.32$, ICH-REHAB: $0.59 \pm 0.08, p=$ 0.553; MdV-ipsi, ICH: $0.07 \pm 0.05$, ICH-REHAB: $0.16 \pm 0.01$, $p=0.072$; Fig. $1 k$ ).

To examine whether the rehabilitation-induced increase in cortico-rubral projections was reflective of a hyperinnervation state, we performed anterograde tracing of the cortico-rubral and cortico-reticular connections in naive rats $(n=4)$. At D12, the number of BDA-positive varicosities touching the red nucleus in naive rats was almost at the midpoint between that of the ICH and ICH-REHAB groups (RNp, $0.28 \pm 0.12, p=0.171 \mathrm{vs} \mathrm{ICH-}$ REHAB; RNm, $0.12 \pm 0.06, p=0.230$ vs ICH-REHAB; Fig. $1 l)$. However, the number of varicosities touching the reticular nuclei in the naive rats was higher than that in the ICH and ICHREHAB groups (Gi-cont, $1.11 \pm 0.33, p=0.002$ vs ICH-REHAB; Gi-ipsi, $0.24 \pm 0.05, p=0.181$ vs ICH-REHAB; MdV-cont, $1.26 \pm 0.30, p<0.001$ vs ICH-REHAB; MdV-ipsi, $0.31 \pm 0.11$, $p=0.262$ vs ICH-REHAB). These data indicate that innervation from the forelimb motor cortex to the red nucleus and the reticular nuclei tended to decrease after capsular stroke, whereas rehabilitative treatment might have reversed this stroke consequence with respect to the cortco-rubral connection. 
a

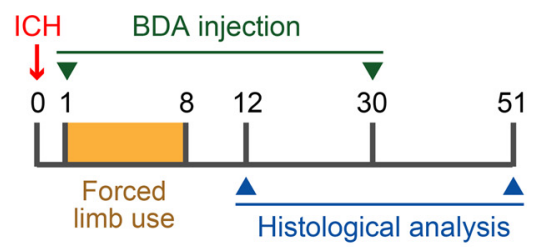

b

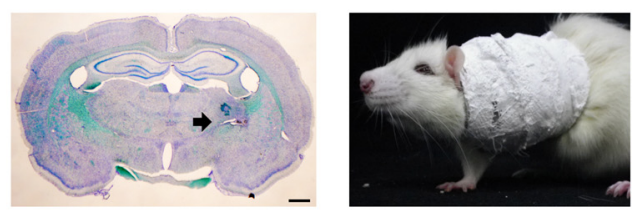

$\mathrm{ICH}$
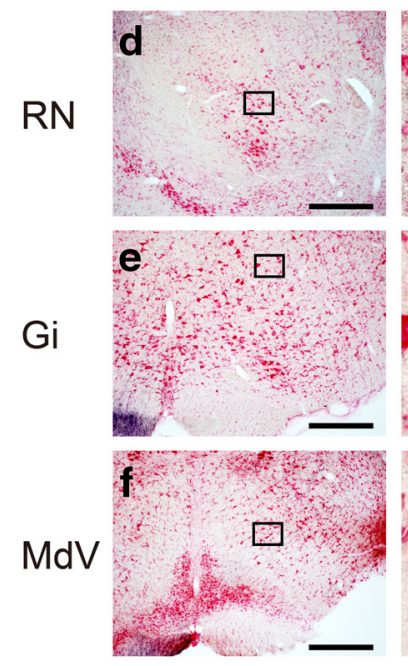

j

D12

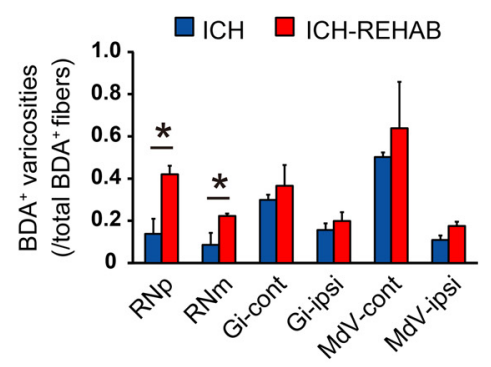

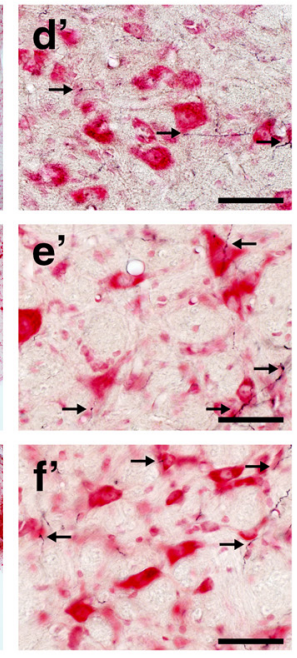

k

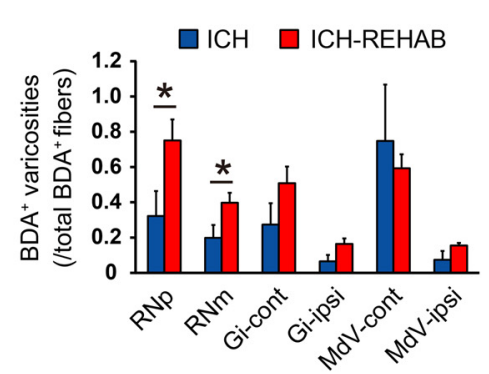

ICH-REHAB
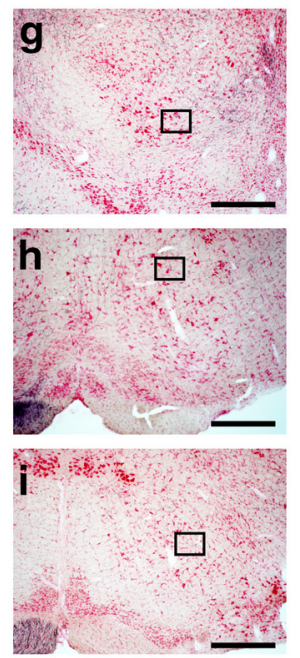

I
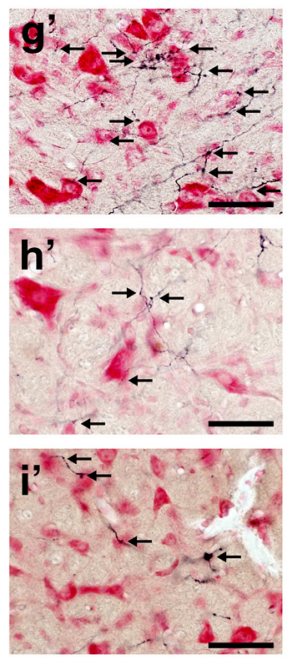

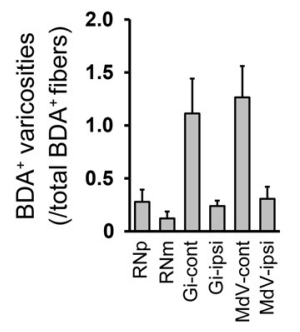

Figure 1. Axonal projections from the forelimb area of the ipsilesional motor cortex to the brainstem in the early and chronic phases of after ICH recovery. $\boldsymbol{a}$, Timeline of the experiments. Forced use of the impaired forelimb was applied on postoperative days 1-8. BDA was injected into the ipsilesional motor cortex on after ICH day 1 or 30, and histological observation was conducted on day 12 or day $51 . \boldsymbol{b}$, Representative Klüver-Barrera-stained image of an ICH. Arrow indicates the hemorrhage site induced by collagenase injection. $\boldsymbol{c}$, Application of forced limb use treatment. A plaster cast restrained the unimpaired forelimb forcing the animal to use the impaired limb in their daily activities. $\boldsymbol{d}$ - $\boldsymbol{i}$, Representative low-magnification images of BDA-labeled fibers in the major brainstem nuclei on day 12 . The sections were counterstained with Neutral Red. $\boldsymbol{d}^{\prime}-\boldsymbol{i} \boldsymbol{i}^{\prime}$, Higher-magnification images of the squared areas in $\boldsymbol{d}-\boldsymbol{i}$. Arrows indicate varicosities apparently contacting neurons. $\boldsymbol{d}, \boldsymbol{g}$, Red nucleus (RN). Compared with the ICH group $\left(\boldsymbol{d}, \boldsymbol{d}^{\prime}\right)$, many BDA-positive fibers were found in the ICH-REHAB group $\left(\boldsymbol{g}, \boldsymbol{g}^{\prime}\right)$. $\boldsymbol{e}, \boldsymbol{h}, \mathrm{Gi} . \boldsymbol{f}, \boldsymbol{i}$, MdV. $\boldsymbol{j}, \boldsymbol{k}$, Quantitative analysis for the varicosities of the cortico-rubral and cortico-reticular axons on day $12(\boldsymbol{j})$ and day $51(\boldsymbol{k})$. The number of BDA-positive varicosities in the parvocellular and magnocellular part of the red nucleus (RNp and RNm) and ipsilateral and contralateral Gi and MdV normalized against the total number of labeled axons in the cerebral peduncle is presented. Data for the number of BDA-positive varicosities in RNp and RNm at day 51 ( $\boldsymbol{k}$ ) are quoted from Ishida et al. (2016). The number of BDA-positive varicosities was significantly higher in the RN in the ICH-REHAB group (D12: $n=6$; D51: $n=7$ ) than in the ICH group (D12: $n=6$; D51: $n=5)$, but not in reticular nuclei (Gi and MDV). $I$, The number of BDA-positive varicosities touching the red nucleus and the reticular nuclei in naive rats $(n=4)$. ${ }^{*} p<0.05$. Data are mean \pm SEM. Scale bars: $\boldsymbol{b}, 1000 \mu \mathrm{m} ; \boldsymbol{d}-\boldsymbol{i}, 500 \mu \mathrm{m} ; \boldsymbol{d}^{\prime}-\boldsymbol{i}^{\prime}, 50 \mu \mathrm{m}$.

The cortico-rubral pathway contributes to rehabilitationinduced functional recovery from the early phase after ICH The tracing experiments suggested that the cortico-rubral projections, not the cortico-reticular projections, were upregulated by force limb use from the early phase at D12 to the chronic phase of recovery at D51. We next investigated whether selective blockade of cortico-rubral tract as early as D12 masks functional recovery by rehabilitation. Viral vectors (FuG-E-TRE-eTeNT.EGFP and AAV-DJ-CMV-rtTAV16) were injected into the red nucleus and the motor cortex, respectively, before ICH lesion and rehabilitation (Fig. 2a), and selective blockade of cortico-rubral tract was performed from the early phase of recovery to confirm direct involvement of the increased cortico-rubral tract in rehabilitation-induced functional recovery. Following injection of the viral vectors and before $\mathrm{ICH}$ lesion and rehabilitation, DOX was administered on days 13-20, and behavioral change was assessed using the skilled reaching task (Fig. 2b,c). 
a

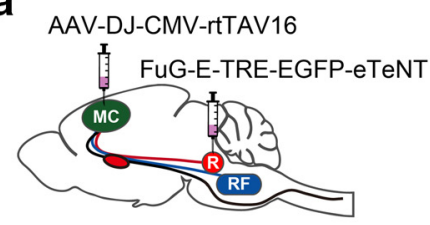

C

pre-DOX (D12) reaching

post-DOX (D14) reaching b

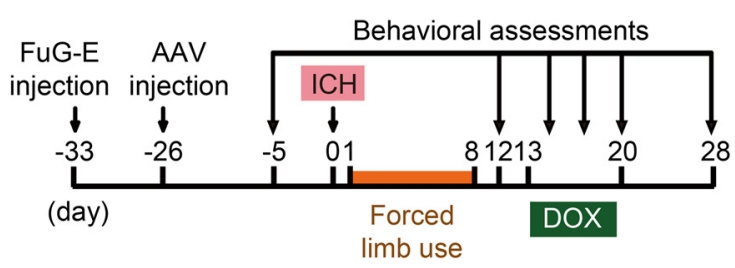

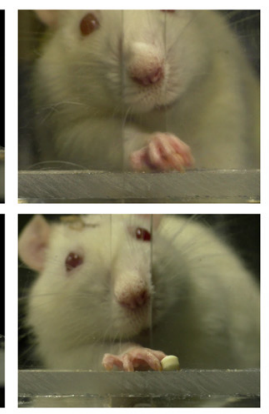

f

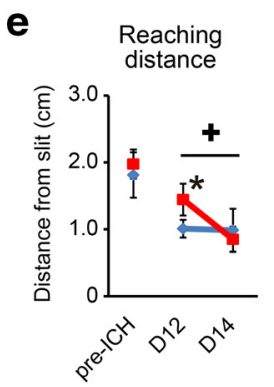

f

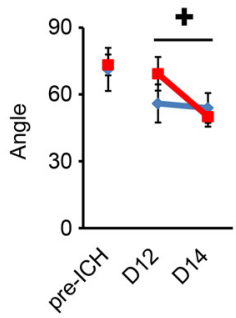

Pronation

g
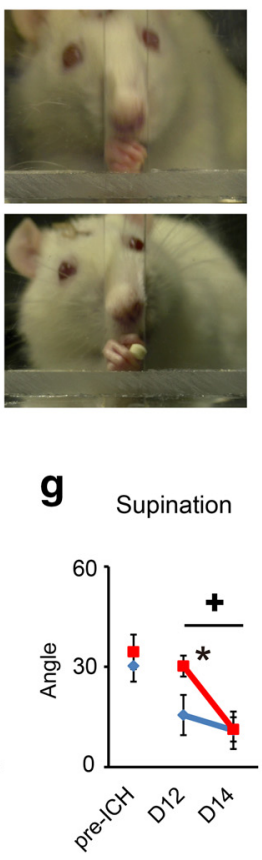

k

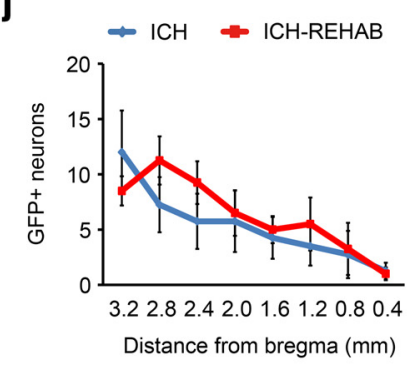

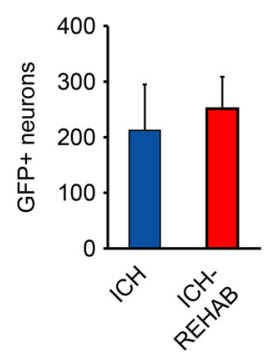

Figure 2. Effects of selective blockade of the cortico-rubral pathway on reaching function in the early-recovery phase. $\boldsymbol{a}$, Schematic diagram of the experimental arrangement. Animals were injected with AAV-DJ-CMV-rtTAV16 into the forelimb area of the ipsilesional motor cortex (MC) and with FuG-E-TRE-EGFP-eTeNT into the ipsilateral red nucleus (R) to block the cortico-rubral tract. $\boldsymbol{b}$, Time course of the experiment. Both FuG-E and AAV vectors were injected before ICH surgery. Behavioral assessments were performed $5 \mathrm{~d}$ before ICH and 12, 14, 17, 20, and 28 d after ICH. DOX was administered on days $13-20$. c, Representative images of the reaching movements of an ICH-REHAB rat before and after DOX administration. DOX-treated rats showed difficulties in extension of their forelimb and rotation of the wrist during grasping. $\boldsymbol{d}$, Average reaching success rates for the ICH $(n=4)$ and ICH-REHAB $(n=5)$ groups. The ICH-REHAB group showed significantly better performance than the ICH group after forced limb use (FLU) at day 12. The recovered performance dropped to the control level during DOX administration. $\boldsymbol{e}-\boldsymbol{g}$, Frame-by-frame analyses of the quality of reaching movements. $\boldsymbol{e}$, Averaged distance of reaching from the front wall of the test apparatus. $\boldsymbol{f}, \boldsymbol{g}$, Averaged range of wrist pronation $(\boldsymbol{f})$ and supination $(\boldsymbol{g})$ during grasping and followed by withdrawal of the limb. $\boldsymbol{h}, \boldsymbol{i}$, Anti-GFP immunohistochemistry showing GFP-positive pyramidal cell bodies in the ipsilesional motor cortex ( $\boldsymbol{h}$ ) and GFP-positive axons in the ipsilesional red nucleus $(\boldsymbol{i})$. $\boldsymbol{i}$, Bottom, Higher-magnification view of the top. Arrows indicate GFP-labeled fibers. $\boldsymbol{j}$, Rostrocaudal distribution of GFP-labeled neurons in ICH $(n=4)$ and ICH-REHAB $(n=4)$ groups. GFP-positive cells were predominantly present in the rostral forelimb area. $\boldsymbol{k}$, Averaged number of total GFP-positive neurons in both groups. ${ }^{*} p<0.05$ versus ICH. ${ }^{+} p<0.05$. Data are mean \pm SEM. Scale bars: $\boldsymbol{i}$, Top, $1000 \mu \mathrm{m} ; \boldsymbol{h}, 500 \mu \mathrm{m} ; \boldsymbol{i}$, Bottom, $100 \mu \mathrm{m}$.

The ICH-REHAB group $(n=5)$ showed a significantly higher reaching success rate compared with the ICH group $(n=4)$ at day 12 (ICH: $15.0 \pm 6.5 \%$, ICH-REHAB: $42.0 \pm 7.8 \%, p=0.038$; Fig. $2 d$ ). DOX administration caused clear impairment of performance to a level comparable with that of the ICH control group (D14, ICH: $20.0 \pm 7.1 \%$, ICH-REHAB: $18.0 \pm 6.0 \%, p=0.835$ ). The performance of ICH-REHAB rats decreased during DOX administration. In contrast, the ICH control group rarely demonstrated behavioral changes after DOX administration. One week after completion of DOX administration, the success rate of pellet retrieval in the ICHREHAB group recovered (51.0 $\pm 8.0 \%$ ). During DOX administration, ICH-REHAB group rats often failed to reach the pellet and displayed abnormal wrist movements to grasp and withdraw a pellet (Fig. 2c).

Further frame-by-frame analysis revealed that the averaged reaching distance of the ICH-REHAB group was significantly 
shortened after DOX administration (D12: $1.44 \pm 0.24 \mathrm{~mm}$, D14: $0.85 \pm 0.19 \mathrm{~mm}, p=0.009$; Fig. $2 e$ ). Similarly, the range of wrist rotation in the ICH-REHAB group was significantly decreased after DOX administration compared with movements before DOX administration (pronation, D12: $69.3 \pm 7.5^{\circ}$, D14: $49.99 \pm 4.5^{\circ}, p=0.012$; Fig. $2 f$; supination, D12: $30.2 \pm 3.1^{\circ}$, D14: $11.3 \pm 3.6^{\circ}, p<0.001$; Fig. $2 g$ ).

Double infection of cortico-rubral tract neurons with viral vectors was verified by immunohistochemistry against GFP, which was tagged with eTeNT. GFP-positive neurons were found in the ipsilesional motor cortex forelimb area (Fig. $2 h$ ), and GFPlabeled fibers were observed around the red nucleus (Fig. 2i). Double-infected cortico-rubral tract neurons were mainly distributed rostral to bregma (Fig. 2j). The number of GFP-labeled neurons was similar between ICH $(n=4)$ and ICH-REHAB $(n=$ 4) groups (ICH: $212.5 \pm 82.4$, ICH-REHAB: $251.3 \pm 57.4, p=$ 0.713; Fig. $2 k$ ).

These data demonstrate that the cortico-rubral tract is directly linked to rehabilitation-induced functional recovery as early as D12 (early-recovery phase after ICH) when significant functional recovery with abundant axonal projections to the red nucleus was caused by intensive limb use at D1-D8.

\section{Blockade of the cortico-rubral pathway during rehabilitation does not disturb functional recovery}

We hypothesized that the cortico-rubral tract would contribute to improvement of impaired forelimb function during rehabilitation after ICH. To test the hypothesis, two viral vectors comprising the Tet-ON system were injected to block the corticorubral tract before $\mathrm{ICH}$, and DOX was administered from the start of forced limb use treatment (Fig. $3 a$ ). In other words, the rats received rehabilitative treatment under cortico-rubral tract blockade.

The ICH-REHAB group $(n=7)$ unexpectedly showed significant functional recovery in the reaching task compared with the ICH group $(n=8)$ at days 12 and 28 , even under continuous DOX administration (D12, ICH: $18.6 \pm 3.3 \%$, ICH-REHAB: $38.3 \pm 1.8 \%, p<0.001$; D28, ICH: $15.0 \pm 2.9 \%$, ICH-REHAB: $55.0 \pm 4.3 \%, p=0.001$; Fig. $3 b$ ). Reaching movements, such as reaching distance and wrist rotation, of the $\mathrm{ICH}-\mathrm{REHAB}$ group did not alter throughout the experiments $(p>0.05$; Fig. $3 c-e)$.

\section{Blockade of the cortico-rubral pathway from the start of rehabilitation induces an alternative increase of the cortico- reticular connection}

To explore the possibility that axonal projections from the motor cortex to the brainstem, other than the cortico-rubral tract, are activated under cortico-rubral tract blockade, we injected BDA into the ipsilesional motor cortex of rats (Fig. $3 a$ ), and the number of BDA-positive varicosities was counted in the Gi and MdV as well as the red nucleus at 3 weeks after the injection. The number of BDA-positive varicosities around the red nucleus was similar between ICH $(n=5)$ and ICH-REHAB $(n=5)$ groups (RNp, ICH: $0.17 \pm 0.02 \%$, ICH-REHAB: $0.24 \pm 0.03 \%, p=$ 0.091; RNm, ICH: $0.05 \pm 0.01 \%$, ICH-REHAB: $0.07 \pm 0.02 \%$, $p=0.363$; Fig. $3 g, h)$. In contrast, a large increase in the number of BDA-positive varicosities around the $\mathrm{Gi}$ and $\mathrm{MdV}$ was found in the ICH-REHAB group $(n=5)$ compared with the ICH group $(n=5)$ (Gi-cont, ICH: $0.48 \pm 0.18$, ICH-REHAB: $1.65 \pm 0.40$, $p=0.039$; Gi-ipsi, ICH: $0.23 \pm 0.11$ ICH-REHAB: $1.10 \pm 0.42$, $p=0.101$; MdV-cont, ICH: $0.74 \pm 0.29$, ICH-REHAB: $2.35 \pm$ 0.57, $p=0.049$; MdV-ipsi, ICH: $0.16 \pm 0.07$, ICH-REHAB: $0.60 \pm 0.14, p=0.033$; Fig. $3 g, h)$. Successful infection of cortico- rubral tract neurons by both viruses was confirmed in both groups by GFP immunoassays (ICH, $n=4: 173.8 \pm 72.8$, ICHREHAB, $n=4: 308.8 \pm 28.1, p=0.135$; Fig. $3 f)$.

The data indicate that the continuous blockade of the corticorubral tract from the start of forced limb use suppressed the increase of the cortico-rubral projections, and suggest the possibility that the cortico-reticular pathway also participated in rehabilitation-induced recovery after ICH.

\section{Silencing the cortico-reticular pathway after rehabilitation does not affect functional recovery after $\mathrm{ICH}$}

To investigate the role of the cortico-reticular tract in rehabilitation-induced functional recovery after $\mathrm{ICH}$, we applied an alternative pathway-selective silencing technique. Using a Credependent double-viral vector approach (FuG-E lentivirus vector and AAV-DJ vector), a silencing DREADD construct $(\mathrm{hM} 4 \mathrm{D}(\mathrm{Gi}))$ was selectively expressed in cortico-reticular tract neurons (Fig. 4a). We first tried to verify the degree of synaptic transmission in the cortico-reticular tract that was blocked by CNO by measuring extracellular LFPs in the caudal reticular formation after contralateral cerebral peduncle stimulation under anesthesia (Fig. $4 b)$. In naive rats $(n=4)$, stimulation of the contralateral cerebral peduncle at $180-200 \mu \mathrm{A}$ induced negative LFP in the MdV with an onset latency of $0.62 \pm 0.04 \mathrm{~ms}$ (Fig. $4 c$ ). The amplitude of the LFP did not appear to change before versus after CNO administration (Fig. 4c). The amplitude of the CDP, which reflects the number of CST axons activated by stimulation of the cerebral peduncle, was also not affected. In contrast, the amplitude of the LFP in the reticular formation had substantially decreased in the DREADD-expressing rats $28 \mathrm{~min}$ after CNO administration (Fig. $4 c, d$ ). The CDP amplitude in this group was not affected. We found that the third LFP amplitude had significantly decreased from 10 to $40 \mathrm{~min}$ after $\mathrm{CNO}$ administration compared with the amplitude at $0 \min (n=8, p<0.05$; Fig. $4 d)$. The largest average reduction in LFP amplitude in the reticular formation was $35.4 \pm 7.7 \%$ (at $28 \mathrm{~min}$ after CNO administration). The cerebral peduncle may include fibers originating from cortical areas other than the vector-injected sites that project to the reticular formation. In addition, we consider the present results to reflect the action of CNO specifically on presynaptic nerve terminals of double-infected cortico-reticular tract axons. Therefore, our results may underestimate the actual effect of $\mathrm{CNO}$ on the transmission from cortico-reticular tract neurons during the behavior. In contrast, the amplitude of the CDP was not affected by $\mathrm{CNO}$ administration ( $p>0.05$; Fig. $4 d$ ). These results demonstrate that the DREADD system expressed by the double viral vector system can selectively silence cortico-reticular tract neurons.

Using this technique, we then aimed to explore whether the cortico-reticular tract was involved in the rehabilitation-induced functional recovery after ICH. Both FuG-E-MSCV-Cre and AAV-DJ-EF1 $\alpha$-DIO-hM4D(Gi)-mCherry vectors were injected before $\mathrm{ICH}$, and $\mathrm{CNO}$ was administered on days 14, 17, and 20 after ICH (Fig. 5a,b). We assessed skilled reaching function 20-40 min after CNO administration because the DREADDinduced silencing of the cortico-reticular tract was prominent during this time window (Fig. 4d). After intensive limb use treatment, the ICH-REHAB group $(n=6)$ showed a significantly higher success rate of pellet retrieval compared with the ICH group $(n=5)$, as before (D12, ICH: $17.0 \pm 2.5 \%$, ICH-REHAB: $45.8 \pm 6.1, p=0.006)$. CNO administration did not affect the success rate of pellet retrieval in either ICH-REHAB or ICH groups (D14, ICH: $18.0 \pm 3.4 \%$, ICH-REHAB: $47.5 \pm 5.9 \%, p=$ 
a

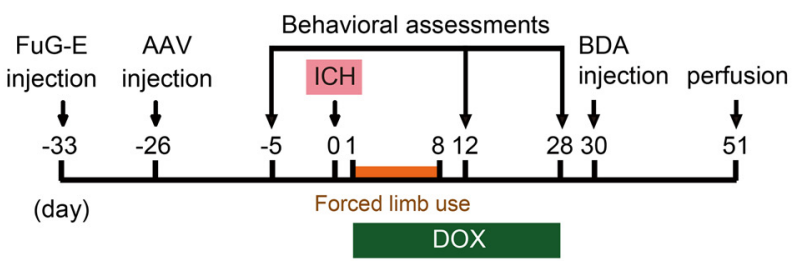

b

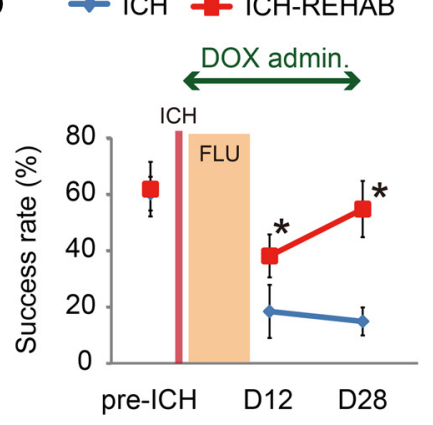

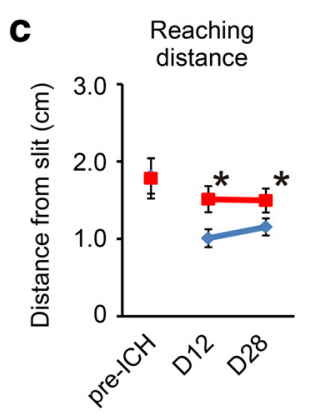
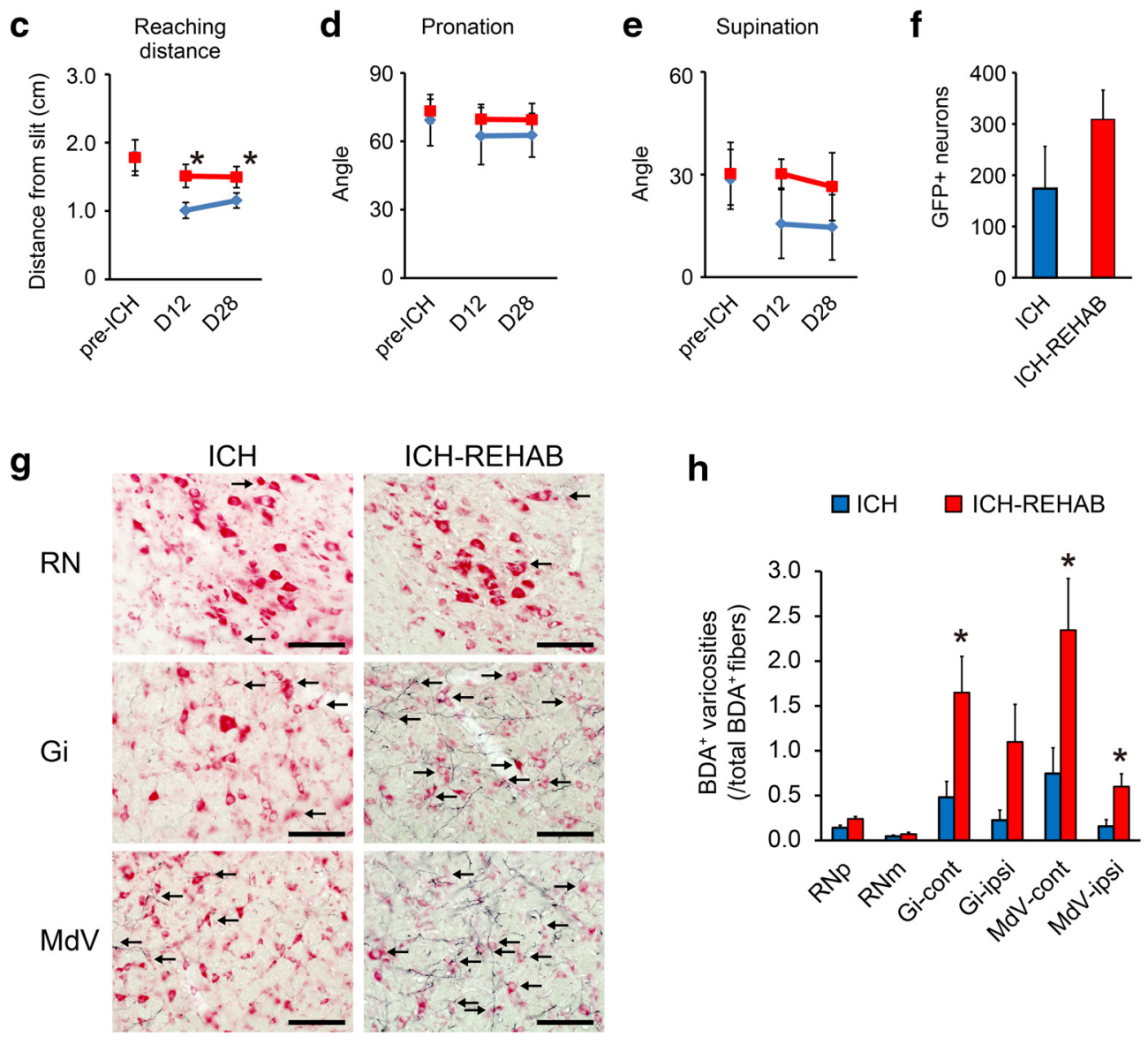

Figure 3. Continuous blockade of the cortico-rubral pathway from the start of intensive limb use treatment. $\boldsymbol{a}$, Timeline of the experiments. Viral vectors were injected before ICH surgery, and DOX administration was conducted from day 1 to day 28 . Behavioral assessments were performed $5 \mathrm{~d}$ before ICH and 12 and $28 \mathrm{~d}$ after ICH. After completion of the behavioral assessments, BDA was injected into the ipsilesional motor cortex to label corticofugal fibers. $\boldsymbol{b}$, Averaged reaching success rate of ICH $(n=7)$ and ICH-REHAB groups $(n=8)$. The ICH-REHAB group showed significantly greater recovery of success rate compared with the ICH group at days 12 and 28. $\boldsymbol{c}-\boldsymbol{e}$, Qualitative analyses of reaching movements. $\boldsymbol{c}$, Averaged reaching distance. $\boldsymbol{d}, \boldsymbol{e}$, Averaged angle of pronation (d) and supination (e) during reaching. No apparent change in the movements was detected throughout the experiment in the ICH-REHAB group. $\boldsymbol{g}$, Representative images of BDA-labeled fibers in brainstem nuclei at day 51 (ICH: $n=5$, ICH-REHAB: $n=5$ ). In RN (top), a few BDA-labeled fibers were observed in both ICH (left column) and ICH-REHAB (right column) groups. In contrast, abundant BDA-positive fibers were found in the ICH-REHAB group in Gi (middle) and MdV (bottom). Arrows indicate varicosities apparently contacting neurons. $\boldsymbol{h}$, Quantitative analysis of the BDA-positive varicosities in the RN and caudal reticular nuclei (Gi and MDV). Intensive limb use-treated animals exhibited a significant increase in BDA-labeled varicosities in contralateral Gi and bilateral MdV under the continuous blockade of the cortico-rubral tract. ${ }^{*} p<0.05$ versus ICH. Data are mean \pm SEM. Scale bar, $100 \mu \mathrm{m}$.

0.003; Fig. 5c). Similarly, reaching distance and wrist rotation during reaching were not affected in the ICH-REHAB group by $\mathrm{CNO}$ administration $(p>0.05$; Fig. $5 d-f)$.

Infection of cortico-reticular tract neurons with both viral vectors was demonstrated by immunohistochemistry against mCherry, as shown in Figure $5 g, h$. Abundant mCherrypositive cells were found in the motor cortex (Fig. $5 g$ ), and labeled fibers were observed in the caudal medullary reticular formation (Fig. 5h). mCherry-positive cortico-reticular tract neurons were also mainly distributed rostral to bregma (Fig. $5 i)$. There was no significant difference in the total number of mCherry-positive cells between ICH $(n=4)$ and ICH-REHAB $(n=4)$ groups (ICH: $2106.3 \pm 445.3$, ICH-REHAB: $2305.0 \pm$ $99.1, p=0.678$; Fig. 5j). Thus, the cortico-reticular tract might not be directly involved in the rehabilitation-induced recovery of forelimb functions. 
a

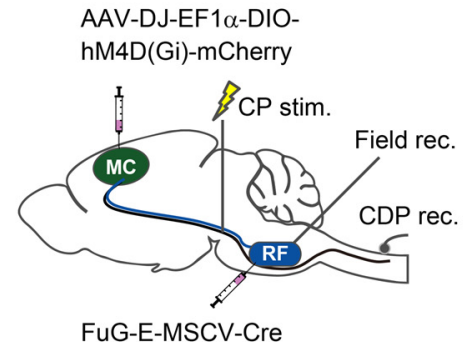

C
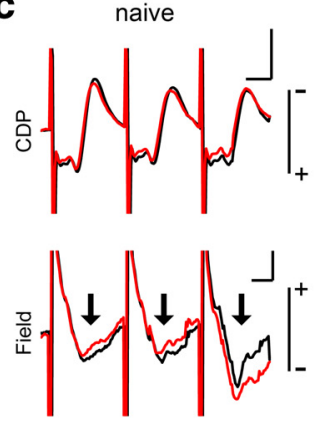

_ pre-CNO admin.
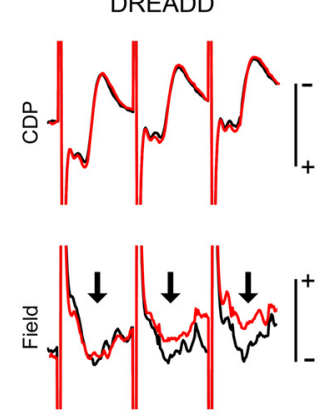

(28 $\mathrm{min})$ b
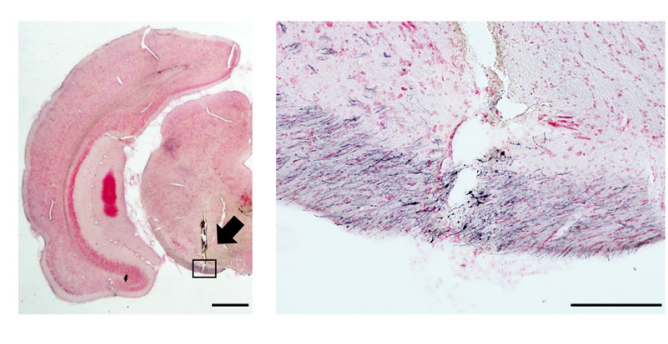

d $\rightarrow$ naive $\quad-$ DREADD

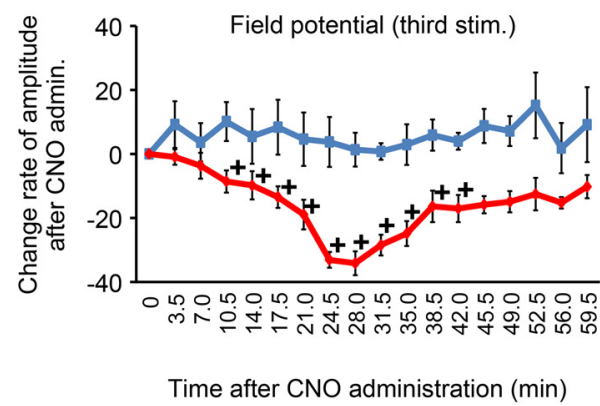

Figure 4. Electrophysiological confirmation of silencing of the cortico-reticular pathway by DREADD. $\boldsymbol{a}$, Depiction of the experiment. Intact animals were injected with AAV-DJ-EF1 $\alpha$-DIOhM4D(Gi)-mCherry into the forelimb area of the ipsilesional motor cortex (MC), and FuG-E-MSCV-Cre was injected into the caudal reticular formation (RF) bilaterally to disturb the cortico-reticular tract. To confirm successful silencing, field potentials (Field) in the MdV and CDP evoked by the stimulation of cerebral peduncle (CP) were recorded under anesthesia. $\boldsymbol{b}$, Anti-mCherry immunohistochemistry of the stimulation site. Arrow indicates the trace of the electrode. Right, Higher magnification of the rectangle in left panel. The stimulation electrode reached the cerebral peduncle containing mCherry-positive fibers.c, Representative field potentials and CDP in a naive rat and in a rat with DREADD-induced silencing at before (black line) and 28 min after CNO administration (red line). Arrows indicate field potentials. Calibration: vertical bar, $1.0 \mathrm{mV}$ for CDP and $0.2 \mathrm{mV}$ for field; horizontal bar, $1 \mathrm{~ms}$ ). $\boldsymbol{d}$, Temporal alteration of LFP after CN0 administration in naive-treated ( $n=$ 4) and DREADD-treated rats $(n=8)$. LFP decreased continuously in the DREADD-treated group from 10 to 40 min after CNO administration, and then recovered. ${ }^{+} p<0.05$ versus 0 min after CNO administration. Data are mean \pm SEM. Scale bars: $\boldsymbol{b}$, Left, $1000 \mu \mathrm{m} ; \boldsymbol{b}$, Right, $200 \mu \mathrm{m}$.

The cortico-reticular pathway becomes essential for functional recovery under blockade of the cortico-rubral pathway during rehabilitation

We hypothesized that the cortico-rubral tract is the first pathway to show a causal relationship to rehabilitation-induced functional recovery after $\mathrm{ICH}$, but that the cortico-reticular tract has the potential to recruit functional recovery in the specific condition, such as the cortico-rubral tract blockade. To test this possibility, we applied the pathway-selective silencing technique to both the cortico-rubral tract and the cortico-reticular tract in the same animals. Four viral vectors were injected in advance of ICH surgery to express eTeNT under the Tet-transactivator system to block the cortico-rubral tract, and to activate the transfected hM4D(Gi) with $\mathrm{CNO}$ to block the cortico-reticular tract (Fig. 6a). In this experiment, the corticorubral tract was blocked from day 1 to day 28 by DOX administration, and the cortico-reticular tract was silenced at days 14, 17, and 20 by $\mathrm{CNO}$ administration (Fig. $6 b$ ).

The success rate of reaching in trained rats was not changed by the single or combined blockade of the cortico-rubral tract or cortico-reticular tract before ICH surgery ( $p>0.05$; Fig. $6 c$ ). Similar to the results in Figure $3 b$, after forced limb use treatment under cortico-rubral tract blockade, the ICH-REHAB group $(n=6)$ showed greater success in the reaching task at day 12 compared with the ICH group $(n=4)(\mathrm{ICH}: 13.8 \pm 2.7 \%$, ICHREHAB: $50.0 \pm 6.9 \%, p=0.006$; Fig. $6 c$ ). After CNO administration, the reaching success rate of the ICH-REHAB group dropped to the same level as that of the ICH control group (D14, ICH: $22.5 \pm 5.2 \%$, ICH-REHAB: $30.0 \pm 3.7 \%, p=0.311$; Fig.
$6 c)$. ICH-REHAB group rats exhibited apparent deficits in reaching in response to $\mathrm{CNO}$ administration (Movies 1,2). Decreased reaching success in the ICH-REHAB group was also observed after the second and third administrations of CNO on days 17 and 20. In addition, their performances were recovered 1 week after the third CNO administration (D28, ICH: $27.5 \pm 3.8 \%$, ICH-REHAB: $55.8 \pm 6.3 \%, p=0.016)$. In contrast, virtually none of the ICH group rats showed an apparent change in reaching success rate after CNO administration $(p>0.05)$.

Qualitative analyses of the reaching movements revealed that the reaching distance of the ICH-REHAB group was shortened significantly in response to CNO administration (D12: $1.45 \pm$ $0.06 \mathrm{~mm}, \mathrm{D} 14$ : $1.08 \pm 0.07 \mathrm{~mm}, p<0.003$; Fig. $6 d$; Movie 1). Although the pronation angle during grasping was not significantly affected by $\mathrm{CNO}$ administration (D12: $62.5 \pm 6.8^{\circ}$, D14: $48.6 \pm 15.2^{\circ}, p=0.243$; Fig. $6 e$ ), the supination angle of the wrist in the reaching movement was significantly decreased in the ICH-REHAB group after CNO administration (D12: $47.4 \pm 6.1^{\circ}$, D14: $17.6 \pm 14.4^{\circ}, p=0.015$; Fig. $6 f$; Movie 2). These data suggest that the cortico-reticular tract becomes responsible for functional recovery induced by rehabilitation in the specific condition of blocked cortico-rubral tract.

Blockade of the cortico-rubral pathway after rehabilitation causes rapid recruitment of the cortico-reticular pathway in functional recovery

We previously showed that impairment of recovered forelimb function by cortico-rubral tract blockade was gradually insensi- 


\section{a}
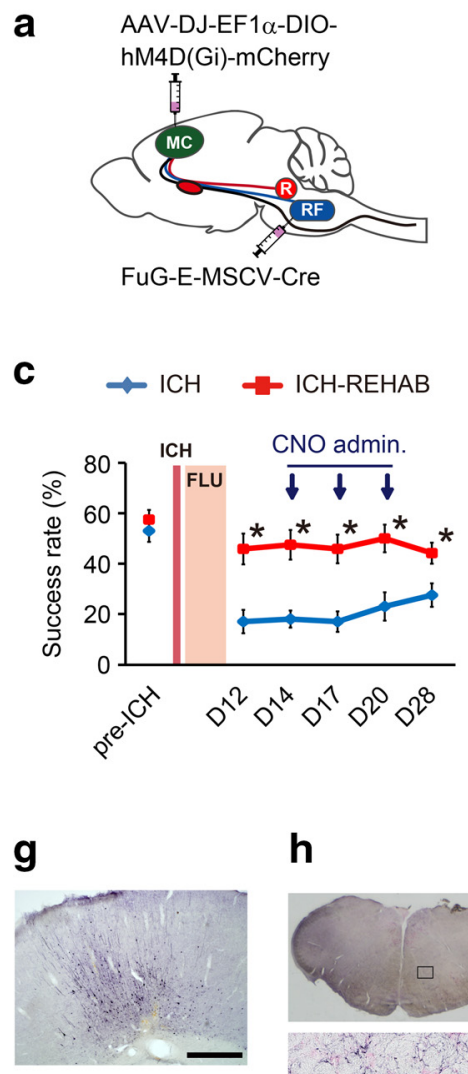

h

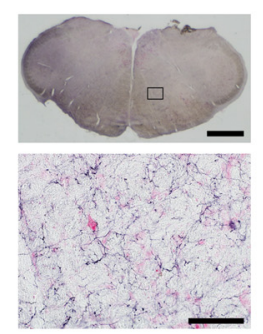

b

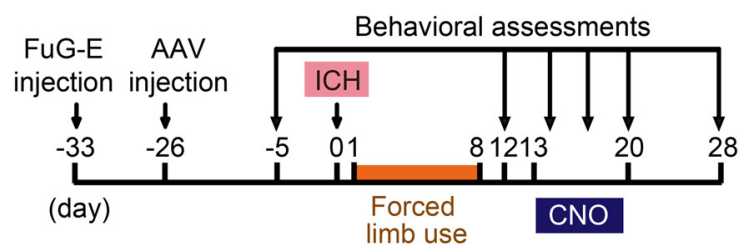

e Pronation

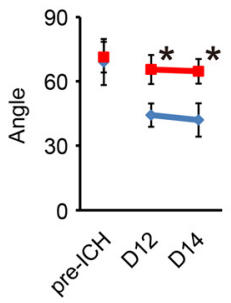

f Supination

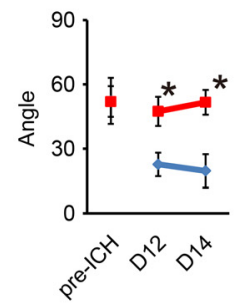

i

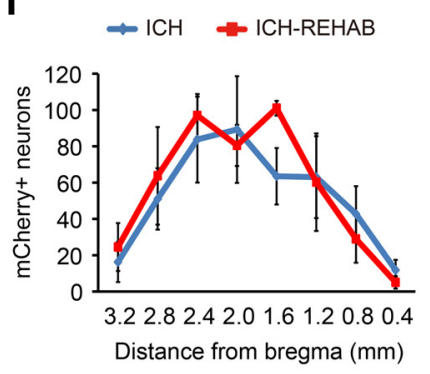

j

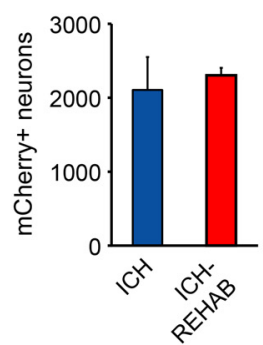

Figure 5. Effects of selective silencing of the cortico-reticular pathway on reaching function in the early-recovery phase. $\boldsymbol{a}$, Depiction of the experiment. Animals were injected with AAV-DJEF1 $\alpha$-DI0-hM4D(Gi)-mCherry into the motor cortex (MC) and with FuG-E-MSCV-Cre into the bilateral reticular formation (RF) to silence the cortico-reticular tract neurons. $\boldsymbol{b}$, Experimental time course. Animals were injected with viral vectors before $\mathrm{I} C \mathrm{H}$, and administered $\mathrm{CN} 0$ on days 14,17 , and 20 . Behavioral assessments were performed $5 \mathrm{~d}$ before $\mathrm{ICH}$ and $12,14,17,20$, and $28 \mathrm{~d}$ after ICH. Each test was conducted within $20-40$ min after $\mathrm{CNO}$ administration. c, Averaged reaching success rate of ICH $(n=5)$ and ICH-REHAB groups $(n=6)$. The ICH-REHAB group showed significant recovery compared with the $\mathrm{ICH}$ group on day 12 . Their performance was not affected by $\mathrm{CNO}$ administration. $\boldsymbol{d}-\boldsymbol{f}$, Detailed analyses of reaching movements. No obvious change was observed in reaching distance $(\boldsymbol{d})$ or in the angle of wrist pronation $(\boldsymbol{e})$ and supination $(\boldsymbol{f}) . \boldsymbol{g}, \boldsymbol{h}$, Anti-mCherry immunohistochemistry. mCherry-positive neurons were found in the ipsilesional motor cortex ( $\boldsymbol{g}$ ), and $\mathrm{mCherry-labeled} \mathrm{fibers} \mathrm{were} \mathrm{prominent} \mathrm{in} \mathrm{the} \mathrm{contralateral} \mathrm{caudal} \mathrm{reticular} \mathrm{nuclei}(\boldsymbol{h}) . \boldsymbol{h}$, Bottom, Higher-magnification view of the top. $\boldsymbol{i}$, Distribution of mCherry-labeled neurons in ICH $(n=$ 4) and ICH-REHAB $(n=4)$ groups along the rostrocaudal axis. $\boldsymbol{j}$, Averaged total number of mCherry-positive neurons of both groups. ${ }^{*} p<0.05$ versus ICH. Data are mean \pm SEM. Scale bars: $\boldsymbol{h}$, Top, $1000 \mu \mathrm{m} ; \boldsymbol{g}, 500 \mu \mathrm{m} ; \boldsymbol{h}$, Bottom, 100.

tive to selective cortico-rubral tract blockade during continuous DOX administration for 1 week (Ishida et al., 2016), which was unexpected. We suggest that this rapid and gradual ineffectiveness of cortico-rubral tract blockade results from the substantial recruitment of the cortico-reticular tract to regain disturbed function. To test this hypothesis, the cortico-rubral tract was blocked for days 13-30, and the cortico-reticular tract was silenced after the recovery of impaired performance by the corticorubral tract blockade (Fig. 7a).

As we reported, the recovered reaching success rate by intensive limb use treatment was decreased by DOX administration (D12, ICH, $n=8: 11.3 \pm 3.0 \%$, ICH-REHAB, $n=9: 37.8 \pm$ $4.0 \%, p<0.001$; D14, ICH: $10.6 \pm 3.6 \%$, ICH-REHAB: $15.0 \pm$ $5.1 \%, p=0.506$; Fig. $7 b$ ). This impairment of recovered reaching function gradually disappeared by day 20 ( $\mathrm{ICH}: 11.9 \pm 3.8 \%$, ICH-REHAB: $45.6 \pm 4.9 \%, p<0.001)$; then CNO was administered on days 21, 24, and 27 (Fig. $7 b$ ).

The reaching success rate in the ICH-REHAB group dropped again after $\mathrm{CNO}$ administration (D21, ICH: $13.8 \pm 5.1 \%$, ICHREHAB: $18.3 \pm 7.3 \%, p=0.623)$. The ICH-REHAB group showed a decreased success rate at the second and third CNO administrations, and their performance recovered $3 \mathrm{~d}$ after the last $\mathrm{CNO}$ administration (D30, ICH: $13.8 \pm 5.5 \%$, ICH-REHAB: $48.0 \pm 4.4 \%, p=0.002)$. Video analyses of reaching movements showed a significant decrease of reaching distance in the ICHREHAB group after DOX administration (D12: $1.62 \pm 0.05$, D14: $1.23 \pm 0.07, p=0.027$; Fig. $7 c$; Movie 3). In addition, the injection of CNO under DOX administration further shortened the distance (D24, $0.85 \pm 0.08, p=0.018$ vs D14). Similarly, the supination angle of the ICH-REHAB group was decreased by DOX administration (D12: $52.3 \pm 8.8^{\circ}$, D14: $31.8 \pm 12.3^{\circ}, p=$ 0.048 ; Fig. $7 e$; Movie 4 ), and was further diminished by additional administration of CNO (D24, $4.8 \pm 6.8^{\circ}, p=0.021$ vs D14). Pronation angle was not significantly changed in either group throughout the experiments ( $p>0.05$; Fig. $7 d$ ).

Double infection of the viral vectors in both cortico-rubral tract and cortico-reticular tract neurons was confirmed by GFP and mCherry immunostaining. Histological analyses revealed that the both GFP- and mCherry-positive neurons were distributed predominantly in the area rostral to bregma (Fig. $7 i, k)$. De- 
a
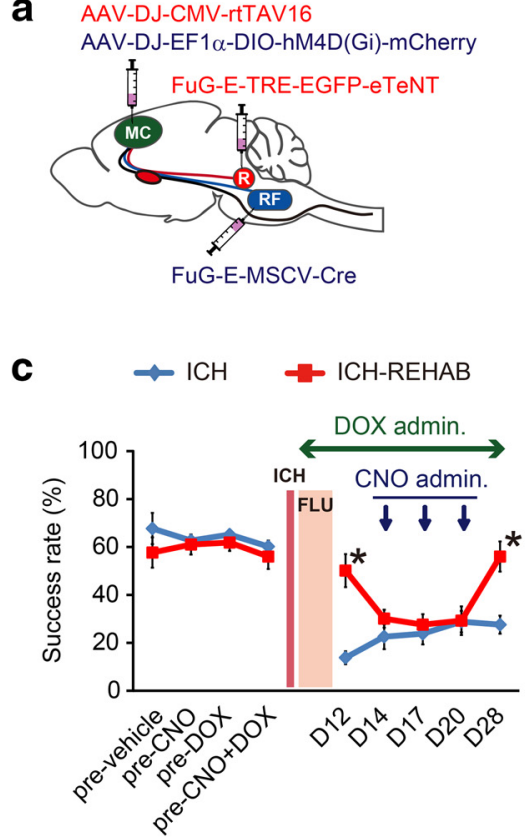

b

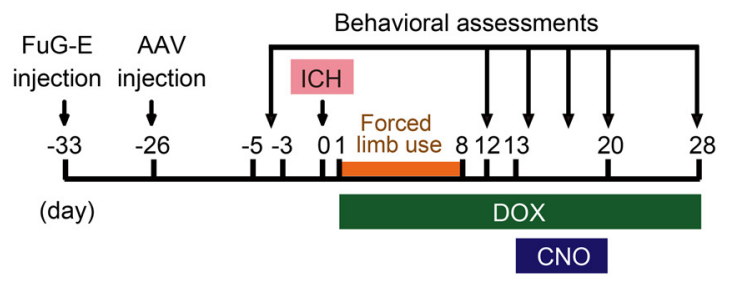

d

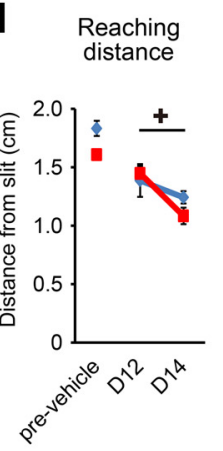

e Pronation $\mathbf{f}$

Supination

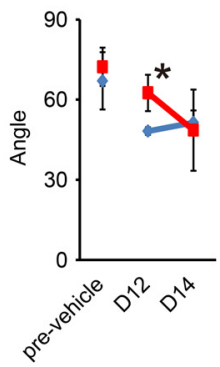

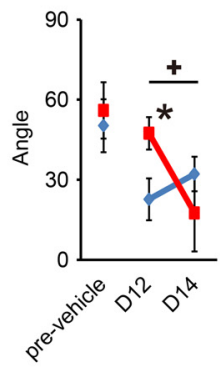

Figure 6. Effects of silencing the cortico-reticular pathway on functional recovery under continuous blockade of the cortico-rubral pathway from the start of rehabilitation. $\boldsymbol{a}$, Experimental design. A mixture of AAV-DJ-CMV-rtTAV16 and AAV-DJ-EF1 $\alpha$-DI0-hM4D(Gi)-mCherry was injected into the ipsilesional motor cortex (MC) and FuG-E-TRE-EGFP-eTeNT and FuG-E-MSCV-Cre were injected into the ipsilateral red nucleus (R) and bilateral reticular formation (RF), respectively, to disturb the function of the cortico-rubral tract and the cortico-reticular tract (cortico-rubral tract) independently. $\boldsymbol{b}$, Experimental time course. Two sets of FuG-E and AAV vectors were injected before ICH surgery. Behavioral assessments were performed before ICH and 12, 14, 17,20, and 28 d after ICH. DOX was administered on days $1-28$, and CNO was injected on days 14,17 , and 20. $C$, Average reaching success rate of ICH $(n=4)$ and ICH-REHAB groups $(n=6)$. The recovered function of the ICH-REHAB group showed significant impairment in response to $\mathrm{CNO}$ administration under the continuous administration of DOX. $\boldsymbol{d}-\boldsymbol{f}$, Average reaching distance (d) and the range of wrist pronation $(\boldsymbol{e})$ and supination $(\boldsymbol{f})$ in the skilled reaching test. Reaching distance and supination angle were significantly decreased in response to CNO administration in DOX-treated ICH-REHAB group rats. ${ }^{*} p<$ 0.05 versus ICH. ${ }^{+} p<0.05$. Data are mean \pm SEM.

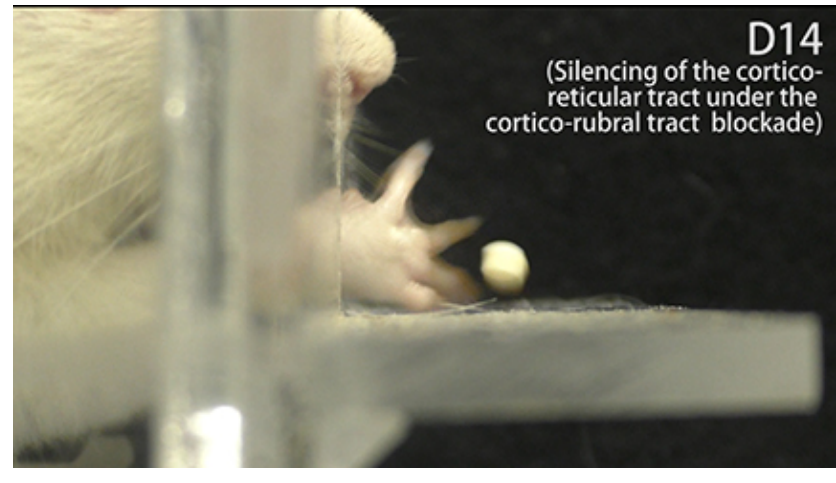

Movie 1. Examples of reaching movement of ICH-REHAB group rats in the experiment to test the recruitment of the cortico-reticular tract under blockade of the cortico-rubral tract after ICH from the start of rehabilitation (Fig. 6). Reaching on $5 \mathrm{~d}$ before and 12 and $14 \mathrm{~d}$ after ICH is shown from a lateral view.

spite the similarity of their distributions, immunofluorescence assays revealed that GFP/mCherry-double-positive neurons were rarely found in the motor cortex $(0.0002 \%$; Fig. $7 f-h)$, indicating that the cortico-rubral tract and cortico-reticular tract originate from different cortical cell populations. Although the total numbers of GFP-labeled neurons were comparable between ICH ( $n=$ $6)$ and ICH-REHAB $(n=6)$ groups (ICH: $170.0 \pm 67.6$, ICHREHAB: $320.0 \pm 92.1, p=0.219$; Fig. $7 j$ ), the number of mCherry-positive neurons had a tendency to be increased in the ICH-REHAB group than the ICH group (ICH: $1369.1 \pm 295.6$, ICH-REHAB: $2755.0 \pm 551.9, p=0.051$; Fig. $7 l$ ). These results

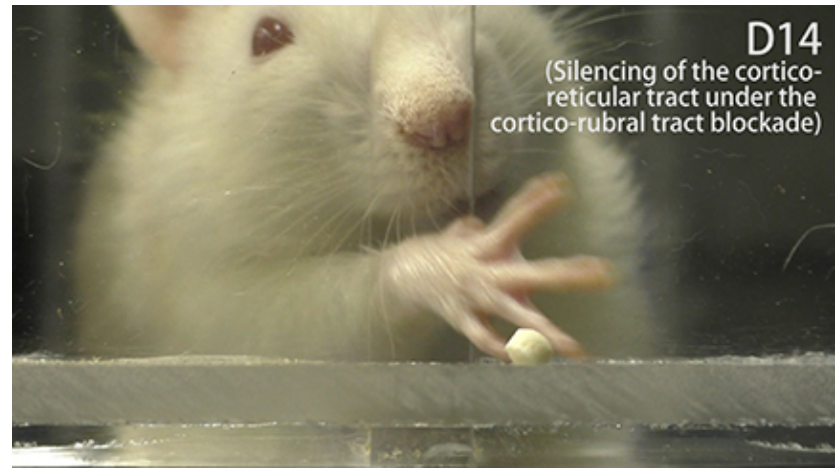

Movie 2. Examples of reaching movement of ICH-REHAB group rats in the experiment to test the recruitment of the cortico-reticular tract under blockade of the cortico-rubral tract from the start of rehabilitation. Reaching on $5 \mathrm{~d}$ before and 12 and $14 \mathrm{~d}$ after ICH is shown from a frontal view.

indicate that the cortico-reticular tract was rapidly recruited and became involved in the control of recovered forelimb function in response to the cortico-rubral tract blockade.

\section{Discussion}

We attempted to determine the timing when the cortico-rubral tract was recruited during rehabilitation (intensive impaired limb use) after ICH, and we also investigated whether the corticoreticular tract could be a substrate in rehabilitation-induced recovery after ICH. Our study clearly demonstrates dynamic involvement of the cortico-brainstem pathways in the process to 
a

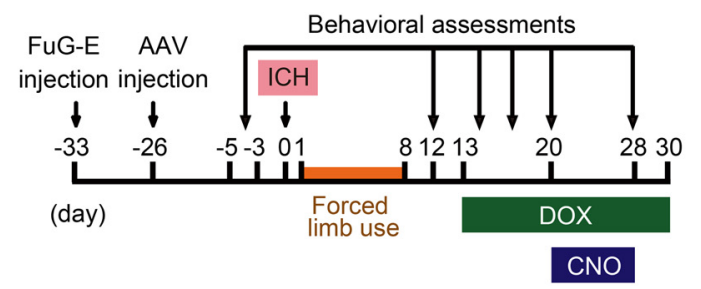

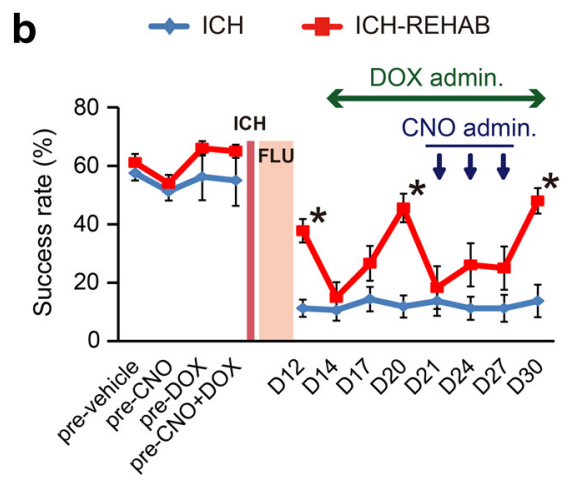

e Supination

C

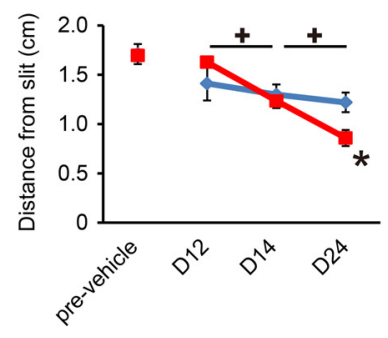

d

Pronation

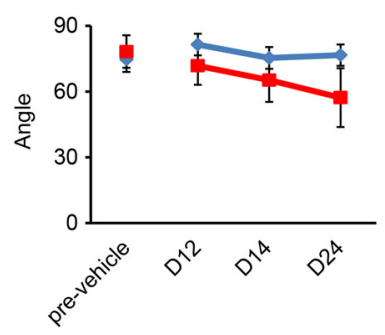

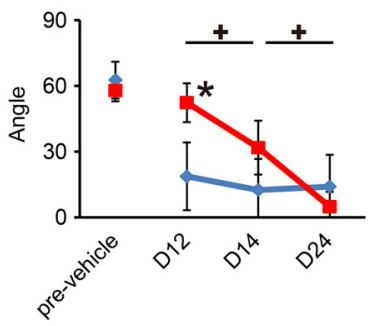
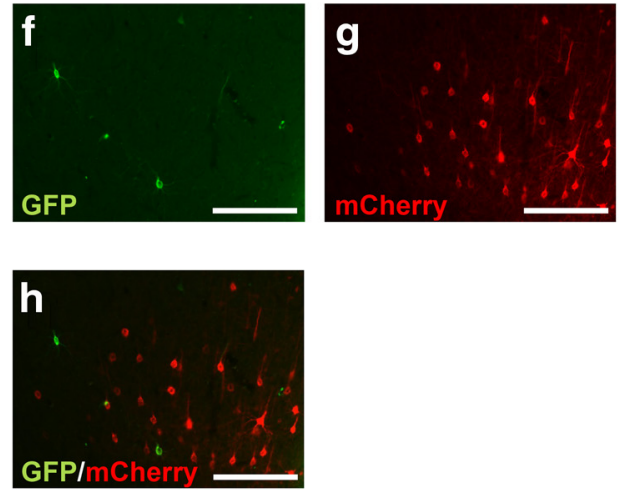
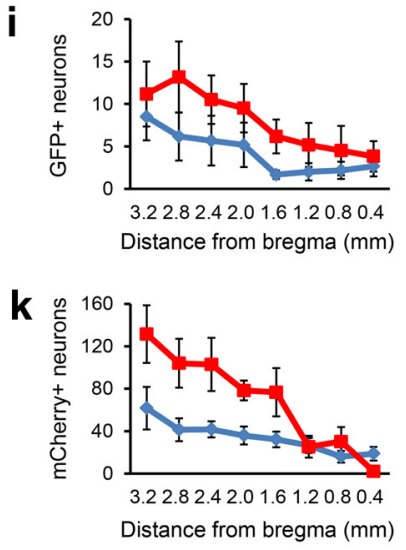

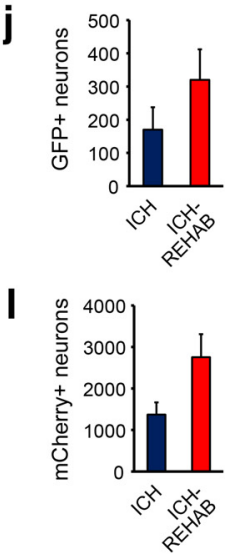

Figure 7. Confirmation of cortico-reticular pathway recruitment in response to blockade of the cortico-rubral pathway after stroke and rehabilitation. $\boldsymbol{a}$, Experimental time course. Animals were injected with two sets of viral vectors before ICH surgery, as shown in Figure 6. Reaching performances were evaluated before ICH to provide a baseline and 12, 14, 17, 20,21, 24, 27, and 30 d after ICH. DOX administration was conducted on days $13-30$, and CNO was injected on days 21,24 , and $27 . \boldsymbol{b}$, Average reaching success rate of ICH $(n=8)$ and ICH-REHAB groups $(n=9)$. The success rate of the ICH-REHAB group fell to the same level as the ICH group after DOX administration but recovered gradually. Additional injection of CNO caused a drastic decrease of the success rate. $\mathbf{c}-\boldsymbol{e}$, Qualitative analyses of the averaged reaching distance $(\boldsymbol{c})$ and the range of wrist pronation $(\boldsymbol{d})$ and supination $(\boldsymbol{e})$. DOX administration caused a significant decrease of reaching distance and supination angle, and additional CNO administration decreased these abilities further. $\boldsymbol{f}-\boldsymbol{h}$, Representative images of GFP-positive $(\boldsymbol{f})$ and mCherry-positive neurons $(\boldsymbol{g})$ in the ipsilesional motor cortex. Few neurons expressed both GFP and mCherry $(\boldsymbol{h}) . \boldsymbol{i}, \boldsymbol{k}$, Distribution of GFP- and mCherry-labeled neurons along the rostrocaudal axis in the ICH $(n=6)$ and ICH-REHAB $(n=6)$ groups. $\boldsymbol{j}, \boldsymbol{I}$, Averaged GFP-positive (i) and mCherry-positive neurons (j) of both groups. ${ }^{*} p<0.05$ versus ICH. ${ }^{+} p<0.05$. Data are mean \pm SEM. Scale bar, $100 \mu \mathrm{m}$.

regain impaired forelimb functions by intensive poststroke rehabilitation. Pathway-selective blockade of the cortico-rubral or the cortico-reticular tract clearly revealed the alternative recruitment of the cortico-reticular pathway for rehabilitationinduced functional recovery when the cortico-rubral pathway failed to function.

Red nucleus neurons involved in skilled reaching project their axons to the cervical cord and innervate the forelimb (Whishaw et al., 1998; Morris et al., 2015). Recently, Rizzi et al. (2019) reported that Vglut2-expressing excitatory rubral cells play an important role in complex fine motor tasks. In addition, Mosberger et al. (2018) demonstrated that axotomized CST neurons extend new axons to the red nucleus and mediate recovered fore- limb function after bilateral pyramidotomy. These findings strongly support the role of the cortico-rubral pathway as an important substrate for the reconstruction of the injured motor system after stroke. In the present study, rehabilitative treatment induced neuronal sprouting in the cortico-rubral tract but not the cortico-reticular tracts in rats subjected to capsular hemorrhage. These results indicate that, compared with cortico-reticular circuits, cortico-rubral connections might be dominantly involved in rehabilitation-induced reorganization for skilled forelimb function, at least in this model.

The caudal reticular nuclei $\mathrm{Gi}$ and $\mathrm{MdV}$ also project their axons to the cervical cord. They are considered to be primarily involved in the control of proximal movements, such as those of 


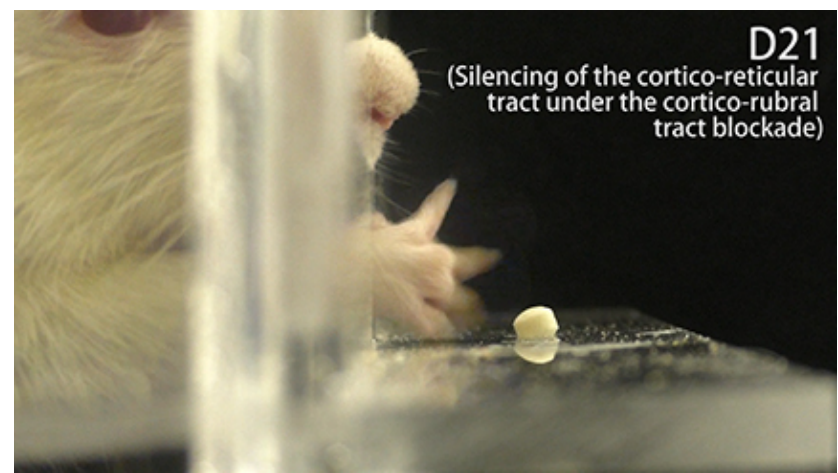

Movie 3. Examples of reaching movement of ICH-REHAB group rats in the experiment to test the rapid recruitment of the cortico-reticular tract in response to blockade of the cortico-rubral tract after rehabilitation (Fig. 7). Reaching on $5 \mathrm{~d}$ before and 12, 14, and $24 \mathrm{~d}$ after ICH is shown from a lateral view.

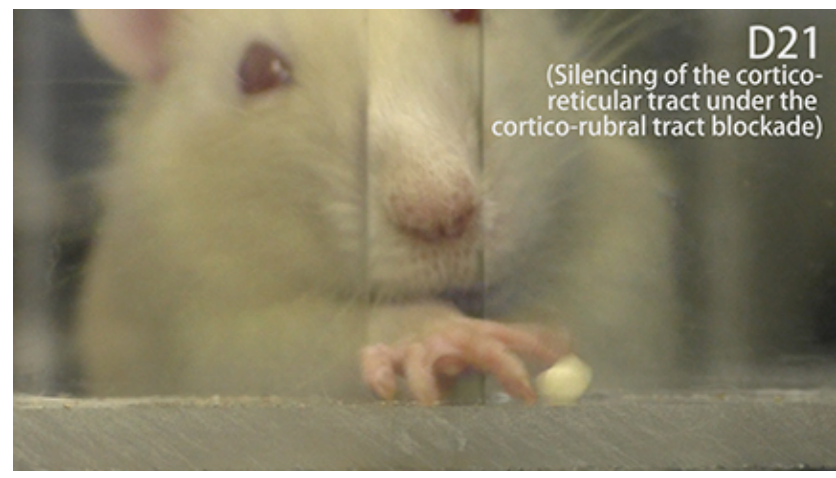

Movie 4. Examples of reaching movement of ICH-REHAB group rats in the experiment to test the rapid recruitment of the cortico-reticular tract in response to blockade of the cortico-rubral tract after rehabilitation. Reaching on $5 \mathrm{~d}$ before and 12,14 , and $24 \mathrm{~d}$ after ICH is shown from a frontal view.

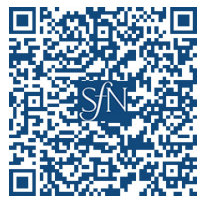

the head and trunk, and to be partly involved in the control of limb movements (Isa and Sasaki, 2002; Schepens and Drew, 2004; Riddle et al., 2009; Baker, 2011). A recent report found that the Gi and $\mathrm{MdV}$ are involved in the skilled control of limb and hand movements, and that they mainly project their axons to spinal motoneurons that innervate proximal and distal forelimb muscles (Esposito et al., 2014). In addition, during rehabilitation, rats with corticospinal and rubrospinal tract injuries exhibited an increase in reticulospinal processes and recovery of reaching function (García-Alías et al., 2015). Together, these findings indicate that the cortico-reticulo-spinal pathway can mediate the effects of poststroke rehabilitation. We revealed a substantial causal relationship between the cortico-reticular tract and functional forelimb recovery only under conditions of continuous corticorubral tract blockade during rehabilitation. We found that this process could take place within a week, indicating that recruitment happens very rapidly. This evidence indicates that the recruitment of circuits by rehabilitation is very dynamic, and that it probably depends on the availability of possible neural substrates. Because the cortical neurons in the cortico-rubral tract are different from those in the cortico-reticular tract, as shown in Figure 7, the mechanism by which the cortico-rubral tract neural circuit switches to that of the cortico-reticular tract remains an interesting topic for future investigation.
Although we focused on plastic changes in cortico-brainstem circuits after ICH and rehabilitation in the present study, other motor-related networks, such as intraspinal circuits, might also be involved in rehabilitation-induced reorganization after stroke. Starkey et al. (2012) reported that the hindlimb motor cortex was involved in forelimb control via neuronal sprouting in the spinal cord after ischemic stroke in the forelimb motor cortex. Wahl et al. (2014) also demonstrated that corticospinal fibers originating in the uninjured side of the forebrain recrossed in the spinal cord for innervation of the impaired forelimb and restored skilled motor function after stroke. Although we did not perform systematic analyses of the alterations of corticofugal fibers in the spinal cord, the plastic changes in the circuits at the level of the spinal cord likely occurred in parallel with the reorganization of cortico-brainstem circuits. Recently, Tohyama et al. (2017) demonstrated that propriospinal neurons play an important role in the recovery of manual dexterity during the early, but not late, postrecovery period after CST lesion. It is possible that multiple circuits, including cortico-brainstem tracts, can be recruited for restoration of impaired function in particular time windows after stroke. Further studies that aim to elucidate the detailed roles and critical time windows for the involvement of motor-related circuits in the process of the poststroke recovery will be important for the development of effective rehabilitation.

In the present study, that the cortico-rubral tract blockade did not affect controls without rehabilitation indicates that intensive limb use training is necessary for recruitment of the corticorubral tract for functional recovery. Recent studies reported that electrical stimulation of the motor cortex promoted axon outgrowth to the brainstem, including red nucleus and spinal targets, and promoted recovery of motor function (Carmel et al., 2013, 2014). We previously demonstrated that intensive impaired limb use induced the upregulation of $\Delta$ FosB protein in the ipsilesional sensorimotor cortex after ICH, indicating rehabilitation-induced repetitive activation of the area (Ishida et al., 2015). These results indicate that poststroke recruitment of compensatory circuits might occur in an activity-dependent manner. In addition, we detected an increased number of cortico-rubral tract axons after forced limb use treatment as early as D12 (early phase), which coincided with functional recovery in the reaching task. In contrast, we previously reported that delayed forced limb use treatment failed to stimulate the motor cortex and promote functional recovery after capsular stroke (Ishida et al., 2015). Upregulation of repair-related molecules and associated cellular changes are known to occur in the acute phase of stroke. Thus, early rehabilitative interventions might be needed for effective recruitment of cortico-brainstem circuits after stroke. However, some basic and clinical studies reported aversive effects of early rehabilitation (Humm et al., 1998; Dromerick et al., 2009). In addition, early high-intensity rehabilitation during the growth phase may induce aberrant fiber patterns and interrupt functional recovery (Wahl et al., 2014). The critical time windows for corticorubral and cortico-reticular plasticity should be considered carefully. Further molecular analysis to identify the mechanisms that induce reorganization of cortico-brainstem circuits is necessary to address this issue.

Although there were a variety of distributions found in different experiments, double-infected cortico-rubral tract or corticoreticular tract neurons were mainly distributed around the rostral region of the forelimb motor area. The rostral region of the forelimb motor cortex in rodents is considered to be homologous to the premotor area in primates (Neafsey and Sievert, 1982; Passingham et al., 1988), and is connected to the brainstem nuclei, 
including the red nucleus and reticular formation in primates (Kuypers and Lawrence, 1967; Monakow et al., 1979). The premotor area is also a key region in recovery after CNS lesion in primates and humans (Liu and Rouiller, 1999; Nishimura et al., 2007; Murata et al., 2015; Schulz et al., 2017). Therefore, reorganization of the cortico-brainstem pathways might be related to premotor area-involved recovery after stroke.

It is known that the rubrospinal and reticulospinal tracts are phylogenetically old and that the structure of the descending tracts is different between rodents and primates. The rubrospinal tract is prominent in lower mammals, including rats, but becomes less evident in higher primates (Massion, 1967). However, the CST has a central role in volitional limb control in primates, especially dexterous finger control (Heffner and Masterton, 1983). Zaaimi et al. (2012) demonstrated recovery of gross motor functions but not the recovery of independent finger movements after injury to the brainstem pyramid in the macaque monkey. Together, it is not simple to apply the present results in rodents to human stroke patients. Recent studies by diffusion tensor MRI imaging revealed strong correlations between fractional anisotropy of the bilateral red nucleus and the level of motor function in chronic stroke patients, indicating possible remodeling of white matter microstructure during recovery (Rüber et al., 2012; Takenobu et al., 2014). Strengthening of the cortico-reticular connections accompanied by recovery of a dexterous hand motor task was demonstrated in monkeys with frontoparietal cortical injury (Darling et al., 2018). Thus, cortico-brainstem-spinal pathways are involved in hand control in primates, including humans. It is also reasonable to assume that the pathways participate in any process to regain impaired functions in human poststroke rehabilitation.

In conclusion, our findings clearly demonstrate the reorganization of cortico-brainstem pathways by intensive rehabilitation after ICH. Using a pathway-selective blockade technique, we demonstrated a direct causal relationship between the corticorubral pathway and rehabilitation-induced functional recovery in the early-recovery phase. In addition, we were able to demonstrate the causality of the alternative cortico-reticular pathway recruitment in the functional recovery by rehabilitation using a combination technique to separately disrupt these corticobrainstem pathways. Further elucidation of the mechanism underlying the reorganization and interaction of these circuits will help the development of more effective rehabilitation strategies after stroke.

\section{References}

Asboth L, Friedli L, Beauparlant J, Martinez-Gonzalez C, Anil S, Rey E, Baud L, Pidpruzhnykova G, Anderson MA, Shkorbatova P, Batti L, Pagès S, Kreider J, Schneider BL, Barraud Q, Courtine G (2018) Corticoreticulo-spinal circuit reorganization enables functional recovery after severe spinal cord contusion. Nat Neurosci 21:576-588.

Bachmann LC, Lindau NT, Felder P, Schwab ME (2014) Sprouting of brainstem-spinal tracts in response to unilateral motor cortex stroke in mice. J Neurosci 34:3378-3389.

Baker SN (2011) The primate reticulospinal tract, hand function and functional recovery. J Physiol 589:5603-5612.

Bareyre FM, Kerschensteiner M, Raineteau O, Mettenleiter TC, Weinmann O, Schwab ME (2004) The injured spinal cord spontaneously forms a new intraspinal circuit in adult rats. Nat Neurosci 7:269-277.

Carmel JB, Kimura H, Berrol LJ, Martin JH (2013) Motor cortex electrical stimulation promotes axon outgrowth to brain stem and spinal targets that control the forelimb impaired by unilateral corticospinal injury. Eur J Neurosci 37:1090-1102.

Carmel JB, Kimura H, Martin JH (2014) Electrical stimulation of motor cortex in the uninjured hemisphere after chronic unilateral injury pro- motes recovery of skilled locomotion through ipsilateral control. J Neurosci 34:462-466.

Darling WG, Ge J, Stilwell-Morecraft KS, Rotella DL, Pizzimenti MA, Morecraft RJ (2018) Hand motor recovery following extensive frontoparietal cortical injury is accompanied by upregulated corticoreticular projections in monkey. J Neurosci 38:6323-6339.

Dromerick AW, Lang CE, Birkenmeier RL, Wagner JM, Miller JP, Videen TO, Powers WJ, Wolf SL, Edwards DF (2009) Very early constraint-induced movement during stroke rehabilitation (VECTORS): a single-center RCT. Neurology 73:195-201.

Esposito MS, Capelli P, Arber S (2014) Brainstem nucleus MdV mediates skilled forelimb motor tasks. Nature 508:351-356.

Fridman EA, Hanakawa T, Chung M, Hummel F, Leiguarda RC, Cohen LG (2004) Reorganization of the human ipsilesional premotor cortex after stroke. Brain 127:747-758.

García-Alías G, Truong K, Shah PK, Roy RR, Edgerton VR (2015) Plasticity of subcortical pathways promote recovery of skilled hand function in rats after corticospinal and rubrospinal tract injuries. Exp Neurol 266:112-119.

Heffner RS, Masterton RB (1983) The role of the corticospinal tract in the evolution of human digital dexterity. Brain Behav Evol 23:165-183.

Humm JL, Kozlowski DA, James DC, Gotts JE, Schallert T (1998) Usedependent exacerbation of brain damage occurs during an early postlesion vulnerable period. Brain Res 783:286-292.

Isa T, Sasaki S (2002) Brainstem control of head movements during orienting: organization of the premotor circuits. Prog Neurobiol 66:205-241.

Ishida A, Tamakoshi K, Hamakawa M, Shimada H, Nakashima H, Masuda T, Hida H, Ishida K (2011) Early onset of forced impaired forelimb use causes recovery of forelimb skilled motor function but no effect on gross sensory-motor function after capsular hemorrhage in rats. Behav Brain Res 225:126-134.

Ishida A, Misumi S, Ueda Y, Shimizu Y, Cha-Gyun J, Tamakoshi K, Ishida K, Hida H (2015) Early constraint-induced movement therapy promotes functional recovery and neuronal plasticity in a subcortical hemorrhage model rat. Behav Brain Res 284:158-166.

Ishida A, Isa K, Umeda T, Kobayashi K, Kobayashi K, Hida H, Isa T (2016) Causal link between the cortico-rubral pathway and functional recovery through forced impaired limb use in rats with stroke. J Neurosci 36:455467.

Kato S, Kobayashi K, Kobayashi K (2014) Improved transduction efficiency of a lentiviral vector for neuron-specific retrograde gene transfer by optimizing the junction of fusion envelope glycoprotein. J Neurosci Methods 227:151-158.

Kinoshita M, Matsui R, Kato S, Hasegawa T, Kasahara H, Isa K, Watakabe A, Yamamori T, Nishimura Y, Alstermark B, Watanabe D, Kobayashi K, Isa $\mathrm{T}$ (2012) Genetic dissection of the circuit for hand dexterity in primates. Nature 487:235-238.

Kobayashi K, Inoue KI, Tanabe S, Kato S, Takada M, Kobayashi K (2017) Pseudotyped lentiviral vectors for retrograde gene delivery into target brain regions. Front Neuroanat 11:65.

Kuypers HG, Lawrence DG (1967) Cortical projections to the red nucleus and the brain stem in the rhesus monkey. Brain Res 4:151-188.

Liu Y, Rouiller EM (1999) Mechanisms of recovery of dexterity following unilateral lesion of the sensorimotor cortex in adult monkeys. Exp Brain Res 128:149-159.

Maier IC, Baumann K, Thallmair M, Weinmann O, Scholl J, Schwab ME (2008) Constraint-induced movement therapy in the adult rat after unilateral corticospinal tract injury. J Neurosci 28:9386-9403.

Massion J (1967) The mammalian red nucleus. Physiol Rev 47:383-436.

Masuda T, Hida H, Kanda Y, Aihara N, Ohta K, Yamada K, Nishino H (2007) Oral administration of metal chelator ameliorates motor dysfunction after a small hemorrhage near the internal capsule in rat. J Neurosci Res $85: 213-222$

Metz GA, Whishaw IQ (2000) Skilled reaching an action pattern: stability in rat (Rattus norvegicus) grasping movements as a function of changing food pellet size. Behav Brain Res 116:111-122.

Monakow KH, Akert K, Künzle H (1979) Projections of precentral and premotor cortex to the red nucleus and other midbrain areas in Macaca fascicularis. Exp Brain Res 34:91-105.

Morris R, Vallester KK, Newton SS, Kearsley AP, Whishaw IQ (2015) The differential contributions of the parvocellular and the magnocellular sub- 
divisions of the red nucleus to skilled reaching in the rat. Neuroscience 295:48-57.

Mosberger AC, Miehlbradt JC, Bjelopoljak N, Schneider MP, Wahl AS, Ineichen BV, Gullo M, Schwab ME (2018) Axotomized corticospinal neurons increase supra-lesional innervation and remain crucial for skilled reaching after bilateral pyramidotomy. Cereb Cortex 28:625-643.

Murata Y, Higo N, Hayashi T, Nishimura Y, Sugiyama Y, Oishi T, Tsukada H, Isa $\mathrm{T}$, Onoe $\mathrm{H}$ (2015) Temporal plasticity involved in recovery from manual dexterity deficit after motor cortex lesion in macaque monkeys. J Neurosci 35:84-95.

Murphy TH, Corbett D (2009) Plasticity during stroke recovery: from synapse to behaviour. Nat Rev Neurosci 10:861-872.

Neafsey EJ, Sievert C (1982) A second forelimb motor area exists in rat frontal cortex. Brain Res 232:151-156.

Nishimura Y, Onoe H, Morichika Y, Perfiliev S, Tsukada H, Isa T (2007) Time-dependent central compensatory mechanisms of finger dexterity after spinal cord injury. Science 318:1150-1155.

Passingham RE, Myers C, Rawlins N, Lightfoot V, Fearn S (1988) Premotor cortex in the rat. Behav Neurosci 102:101-109.

Peterson BW, Pitts NG, Fukushima K (1979) Reticulospinal connections with limb and axial motoneurons. Exp Brain Res 36:1-20.

Riddle CN, Edgley SA, Baker SN (2009) Direct and indirect connections with upper limb motoneurons from the primate reticulospinal tract. J Neurosci 29:4993-4999.

Rizzi G, Coban M, Tan KR (2019) Excitatory rubral cells encode the acquisition of novel complex motor tasks. Nat Commun 10:2241.

Rüber T, Schlaug G, Lindenberg R (2012) Compensatory role of the corticorubro-spinal tract in motor recovery after stroke. Neurology 79:515-522.

Schepens B, Drew T (2004) Independent and convergent signals from the pontomedullary reticular formation contribute to the control of posture and movement during reaching in the cat. J Neurophysiol 92:2217-2238.
Schulz R, Park E, Lee J, Chang WH, Lee A, Kim YH, Hummel FC (2017) Interactions between the corticospinal tract and premotor-motor pathways for residual motor output after stroke. Stroke 48:2805-2811.

Starkey ML, Bleul C, Zörner B, Lindau NT, Mueggler T, Rudin M, Schwab ME (2012) Back seat driving: hindlimb corticospinal neurons assume forelimb control following ischaemic stroke. Brain 135:3265-3281.

Takenobu Y, Hayashi T, Moriwaki H, Nagatsuka K, Naritomi H, Fukuyama $\mathrm{H}$ (2014) Motor recovery and microstructural change in rubro-spinal tract in subcortical stroke. Neuroimage Clin 4:201-208.

Taub E, Uswatte G, Elbert T (2002) New treatments in neurorehabilitation founded on basic research. Nat Rev Neurosci 3:228-236.

Tohyama T, Kinoshita M, Kobayashi K, Isa K, Watanabe D, Kobayashi K,Liu M, Isa T (2017) Contribution of propriospinal neurons to recovery of hand dexterity after corticospinal tract lesions in monkeys. Proc Natl Acad Sci U S A 114:604-609.

Wahl AS, Omlor W, Rubio JC, Chen JL, Zheng H, Schröter A, Gullo M, Weinmann O, Kobayashi K, Helmchen F, Ommer B, Schwab ME (2014) Neuronal repair: asynchronous therapy restores motor control by rewiring of the rat corticospinal tract after stroke. Science 344:1250-1255.

Whishaw IQ, Gorny B, Sarna J (1998) Paw and limb use in skilled and spontaneous reaching after pyramidal tract, red nucleus and combined lesions in the rat: behavioral and anatomical dissociations. Behav Brain Res 93:167-183.

Zaaimi B, Edgley SA, Soteropoulos DS, Baker SN (2012) Changes in descending motor pathway connectivity after corticospinal tract lesion in macaque monkey. Brain 135:2277-2289.

Z'Graggen WJ, Metz GA, Kartje GL, Thallmair M, Schwab ME (1998) Functional recovery and enhanced corticofugal plasticity after unilateral pyramidal tract lesion and blockade of myelin-associated neurite growth inhibitors in adult rats. J Neurosci 18:4744-4757. 